ARTICLE

DOI: $10.1038 / \mathrm{s} 41467-018-05933-8$

\title{
LincRNA H19 protects from dietary obesity by constraining expression of monoallelic genes in brown fat
}

\author{
Elena Schmidt (10 1,2, Ines Dhaouadi,2, Isabella Gaziano ${ }^{1,2}$, Matteo Oliverio (1) 1,2, Paul Klemm², \\ Motoharu Awazawa 1,2, Gerfried Mitterer ${ }^{3}$, Eduardo Fernandez-Rebollo4,5, Marta Pradas-Juni,2,4, \\ Wolfgang Wagner (10 5, Philipp Hammerschmidt1,2, Rute Loureiro ${ }^{1,2,4}$, Christoph Kiefer ${ }^{4}$, Nils R. Hansmeier ${ }^{1,2}$, \\ Sajjad Khani ${ }^{1,2}$, Matteo Bergami ${ }^{2}$, Markus Heine ${ }^{6}$, Evgenia Ntini ${ }^{7}$, Peter Frommolt ${ }^{2}$, Peter Zentis ${ }^{2}$, \\ Ulf Andersson Ørom (1) ${ }^{8}$, Jörg Heeren ${ }^{6}$, Matthias Blüher ${ }^{9}$, Martin Bilbann ${ }^{3,10}$ \& Jan-Wilhelm Kornfeld (1) 1,2,4
}

Increasing brown adipose tissue (BAT) thermogenesis in mice and humans improves metabolic health and understanding BAT function is of interest for novel approaches to counteract obesity. The role of long noncoding RNAs (IncRNAs) in these processes remains elusive. We observed maternally expressed, imprinted IncRNA H19 increased upon coldactivation and decreased in obesity in BAT. Inverse correlations of $\mathrm{H} 19$ with BMI were also observed in humans. H19 overexpression promoted, while silencing of H19 impaired adipogenesis, oxidative metabolism and mitochondrial respiration in brown but not white adipocytes. In vivo, H19 overexpression protected against DIO, improved insulin sensitivity and mitochondrial biogenesis, whereas fat H19 loss sensitized towards HFD weight gains. Strikingly, paternally expressed genes (PEG) were largely absent from BAT and we demonstrated that $H 19$ recruits PEG-inactivating H19-MBD1 complexes and acts as BAT-selective PEG gatekeeper. This has implications for our understanding how monoallelic gene expression affects metabolism in rodents and, potentially, humans.

\footnotetext{
${ }^{1}$ Max Planck Institute for Metabolism Research, Gleueler Strasse 50, 50931 Köln, Germany. ${ }^{2}$ Cologne Cluster of Excellence: Cellular Stress Responses in Ageing-associated Diseases (CECAD), Joseph-Stelzmann-Str. 26, 50931 Köln, Germany. ${ }^{3}$ Department of Laboratory Medicine, Medical University of Vienna, 1090 Vienna, Austria. ${ }^{4}$ Department for Biochemistry and Molecular Biology (BMB), University of Southern Denmark, Campusvej 55, 5230 Odense M, Denmark. ${ }^{5}$ Helmholtz-Institute for Biomedical Engineering, Stem Cell Biology and Cellular Engineering, RWTH Aachen University Medical School, Pauwelstrasse 20, 52074 Aachen, Germany. ${ }^{6}$ Institute for Biochemistry and Molecular Cell Biology, Martinistraße 52,20246 Hamburg, Germany. ${ }^{7}$ Max Planck Institute for Molecular Genetics, Innestrasse 63-73, 14195 Berlin, Germany. ${ }^{8}$ Institute for Molecular Biology and Genetics, Aarhus University, C F Møllers Alle 3, 8000 Aarhus C, Denmark. ${ }^{9}$ Department of Medicine, University of Leipzig, 04103 Leipzig, Germany. ${ }^{10}$ Core Facilities, Medical University of Vienna, 1090 Vienna, Austria. These authors contributed equally: Martin Bilban, Jan-Wilhelm Kornfeld. Correspondence and requests for materials should be addressed to M.B. (email: martin.bilban@meduniwien.ac.at) or to J.-W.K. (email: janwilhelmkornfeld@bmb.sdu.dk)
} 
O besity results from chronic imbalances between caloric intake and energy expenditure (EE), which finally culminates in development of cardiometabolic complications $^{1}$, artherosclerosis, ${ }^{2}$ liver manifestations of metabolic disease $^{3}$, and type 2 diabetes (T2D) ${ }^{1}$. In contrast to white adipose tissue (WAT), which primarily stores lipids, brown adipose tissue (BAT) represents a specialized organ that supports energy catabolism by converting dietary and stored nutrients like lipids and glucose into heat (non-shivering thermogenesis) by virtue of uncoupling the electrochemical energies stored in proton gradients across mitochondrial membranes ${ }^{4}$. The (re)-discovery of functional BAT in (adult) humans ${ }^{5}$, the observation that surgical and genetic ablation of BAT sensitizes towards obesity in mice ${ }^{6}$ and the clinically relevant fact that reductions in ambient temperatures improve metabolism in lean $^{7}$ and diabetic ${ }^{8}$ subjects, spurred great interest in harnessing the catabolic properties of BAT for therapeutic purposes. In recent years, significant progress was made in understanding and functionally dissecting the transcriptional and epigenetic control of BAT differentiation and function by protein-coding genes ${ }^{9}$. Yet, large-scale sequencing initiatives such as ENCODE ${ }^{10}$ demonstrated that RNA transcription is not confined to protein-coding genes but constitutes a pervasive phenomenon observed throughout the majority of genomes in higher organisms ${ }^{11}$, giving rise to thousands of small noncoding RNAs like microRNAs but also long noncoding RNAs (lncRNAs). LncRNAs are (arbitrarily) defined as transcripts $\geq 200$ nt with low species conservation at the nucleotide level, low coding potentials, and simplistic gene architectures ${ }^{12}$. Although lncRNA numbers are debated, RNA-Seq meta-analyses in human tissues identified $>60,000$ lncRNAs, which surpassed the ca. 22,000 coding genes detected in the same study ${ }^{13}$.

Despite progress in identifying and functionally dissecting lncRNAs involved in cellular and organismal ageing ${ }^{14}$, metabolic homeostasis $^{15}$, and adipose tissue biology ${ }^{16,17}$, our understanding of how lncRNA control adipogenesis and BAT differentiation and function in particular remains elusive. To gain molecular insights into these processes, we here performed RNA-Sequencing (RNASeq) in BAT of mice exposed cold or exposed to chronic high-fat diet feeding and observed that monoallelically expressed (imprinted) lncRNA H19 correlated with BAT activation in mouse, but also humans. In vitro and in vivo gene manipulation identified BAT-selective roles for H19 in controlling adipocyte differentiation and function and systemic energy metabolism. Crucially, we found that many adipose-selective imprinted genes expressed from paternal alleles were absent from BAT and could demonstrate that $H 19$ forms H19-MBD1 chromatin modifier complexes that specifically repress paternally expressed imprinted genes in brown, not white, adipocytes, thereby serving as selective PEG gatekeeper in BAT.

\section{Results}

RNA-sequencing for BAT-regulatory lncRNAs. To identify mRNAs and lncRNAs correlating with BAT function, we exposed lean C57BL/6 mice to thermal stress $\left(4^{\circ} \mathrm{C}\right.$ for $24 \mathrm{~h}$ ) compared to $22^{\circ} \mathrm{C}$ housed mice or to chronic high-fat-diet (HFD) compared to micronutrient-matched control diet (CD) feeding. Next, we performed RNA-Sequencing (RNA-Seq) in these BAT samples and observed 1394/433 upregulated and 1147/429 downregulated mRNAs together with 71/6 upregulated and 101/33 downregulated lncRNAs across cold/DIO conditions (fold-change $\geq 2$ or $\leq-2$; Fig. 1a-d, Supplementary Data 1,2). Alterations in BAT thermogenesis were confirmed by elevated and decreased Uncoupling Protein 1 (Ucp1) and ELOVL Fatty Acid Elongase 3 (Elovl3) mRNA expression during cold or diet-induced obesity (DIO), respectively (Fig. 1e, f). Due to its BAT-selective expression-as for Ucp1 and Elovl3 (Fig. 1e-g)-and strong eutherian sequence conservation, we focussed on the intergenic lncRNA (lincRNA) H19, which was induced in cold-exposed and decreased in obese BAT (Fig. 1g). Notably, H19 expression was not altered accordingly in subcutaneous (scWAT) or visceral (vWAT) white adipose depots in contrast to browning/beiging markers like Ucp1 (Fig. 1h-j), arguing for a selective involvement of $\mathrm{H} 19$ in brown but not beige adipocyte differentiation and function.

H19 controls brown adipogenesis and BAT oxidative metabolism. When performing locked nucleic acid (LNA)-mediated RNA interference (RNAi) in stromal-vascular fraction (SVF) adipocyte precursor cells isolated from three major adipose depots (BAT, scWAT, and vWAT), we observed blunted lipid accumulation, reduced Oil Red O (ORO) lipid staining, and impaired expression of BAT markers like Cell Death-Inducing DFFA-Like Effector A (Cidea), Iodothyronine Deiodinase 2 (Dio2), and Ucp 1, as well as reduced UCP1 and PPARG Coactivator 1 Alpha (PGC1A) protein levels, together with common adipose tissue markers like Adiponectin (Adipoq), CCAAT/Enhancer Binding Protein Alpha (Cebpa), Fatty Acid Binding Protein 4 (Fabp4), and Peroxisome Proliferator Activated Receptor Gamma (Pparg) upon H19 RNAi in primary adipocyte precursor cells. We thus concluded that $\mathrm{H} 19$ is required for the commitment of BAT but not scWAT or vWAT-derived progenitors (Fig. 2a-c).

Next, we aimed to dissect the cell-intrinsic and metaboregulatory properties of $\mathrm{H} 19$ using in vitro gain/loss-of-function approaches. Cellular bioenergetics analyses using Seahorse technology confirmed and calculation of coupling efficiencies further supported that H19 RNAi impaired (Fig. 2d, Supplementary Fig. 1a, c), whilst H19 overexpression supported oxidative metabolism, uncoupling and extracellular acidification rates (ECAR) in differentiated brown adipocytes (Supplementary Fig. 1b, d, f) and sensitized brown adipocytes to stimulation with beta3-adrenoreceptor agonist CL316,243 (Supplementary Fig. 1e). This illustrates that $H 19$ supports adipocyte differentiation but also thermogenic gene expression, oxidative metabolism and mitochondrial dynamics in mature adipocytes in a BATspecific manner. Interestingly, glycolysis was unaltered in H19 overexpressing adipocytes (Supplementary Fig. 1g), pinpointing towards selective roles of $\mathrm{H} 19$ in mitochondrial lipid oxidation not carbohydrate metabolism.

H19 was reported to reside in nuclear, but also cytoplasmic and ribosomal fractions in other cell types ${ }^{11,18}$. As LNA-mediated RNAi acts via RNAse $\mathrm{H}$-mediated transcript decay predominantly in nuclei, we next targeted H19 using small interfering RNAs (siRNAs), which act preferentially in the cytoplasm. We observed H19 loss across subcellular compartments using both techniques (Supplementary Fig. 1h, i). Doing so, we confirmed critical roles for $\mathrm{H} 19$ in commitment of brown and subcutaneous ${ }^{19}$ adipocytes as demonstrated by decreased ORO staining (Fig. 2f-h) and reduced expression of brown and white adipogenesis markers including Pgcla (Fig. 2i). Expression of adipocyte precursor genes like Delta Like Non-Canonical Notch Ligand 1 (Dlk1), Runt Related Transcription Factor 2 (Runx2), Platelet Derived Growth Factor Receptor Alpha (Pdgfra), GATA Binding Protein 2 (Gata2), Lymphocyte Antigen 6 Family Member E (Ly6a), and Transforming Growth Factor Beta 2 $(T g f b 2)$ remained high in BAT, but not scWAT or vWAT-derived mature adipocytes upon LNA/siRNA-mediated H19 silencing (Supplementary Fig. 2a, b). Independent H19 LNA and siRNA inhibitors evoked similar arrests in brown adipocyte differentiation and impaired expression of BAT markers (Supplementary Fig. 2c, d). 
H19 was historically discovered as monoallelically expressed gene in which solely maternal alleles express H19 in an epigenetic process termed genomic imprinting. Here, the reciprocally expressed H19-Igf2 imprinted gene cluster critically depends on the enhancer-blocking properties of CCCTC-Binding Factor (Ctcf) proteins $^{20,21}$. As no defects in BAT adipogenesis occurred after Ctcf RNAi (Supplementary Fig. 2e) and because BAT Ctcf and
Igf2 levels were not affected by DIO or cold (Supplementary Fig. 2f, g), we reasoned that cold/DIO-evoked alterations in H19 are not secondary to altered H19-Igf2 imprinting, corroborating earlier reports about reduced $H 19$ expression independent from traditional H19-Igf2 imprinting control ${ }^{22}$. Further, the H19encoded microRNA miR-675 was not expressed in BAT (Supplementary Fig. 2h, i), arguing against the involvement of a

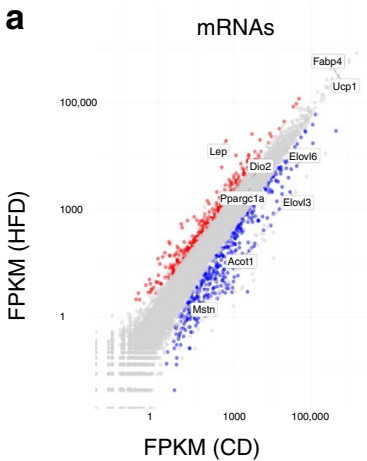

C

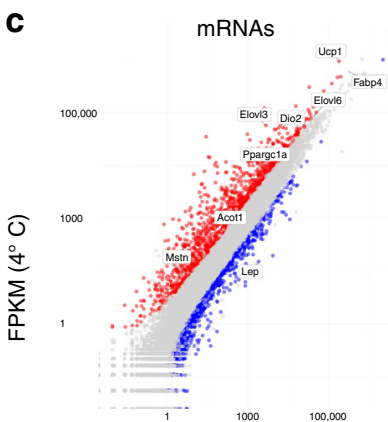

$\operatorname{FPKM}\left(22^{\circ} \mathrm{C}\right)$ b

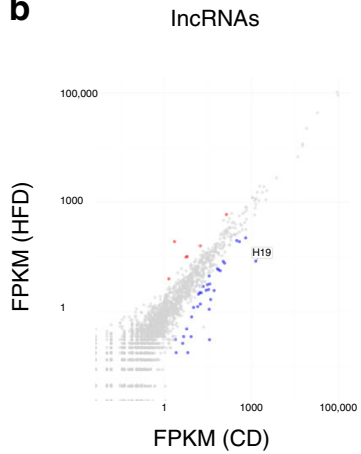

d

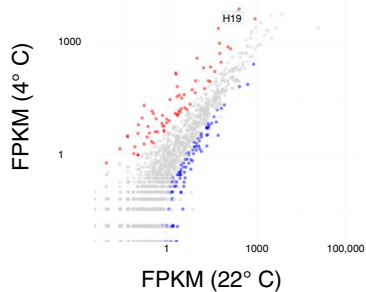

Fold-change $\geq 2$

Fold-change $\leq 2, \geq-2$

Fold-change $\leq-2$
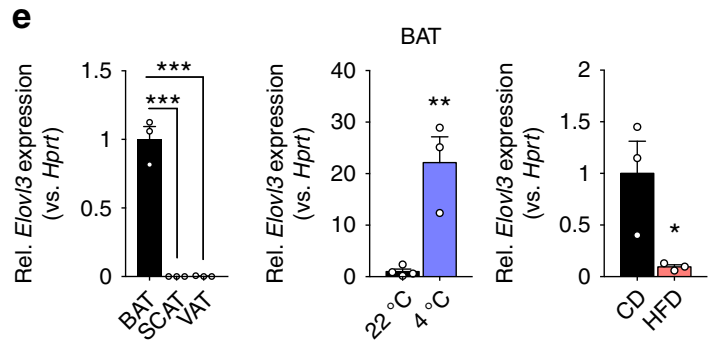

h
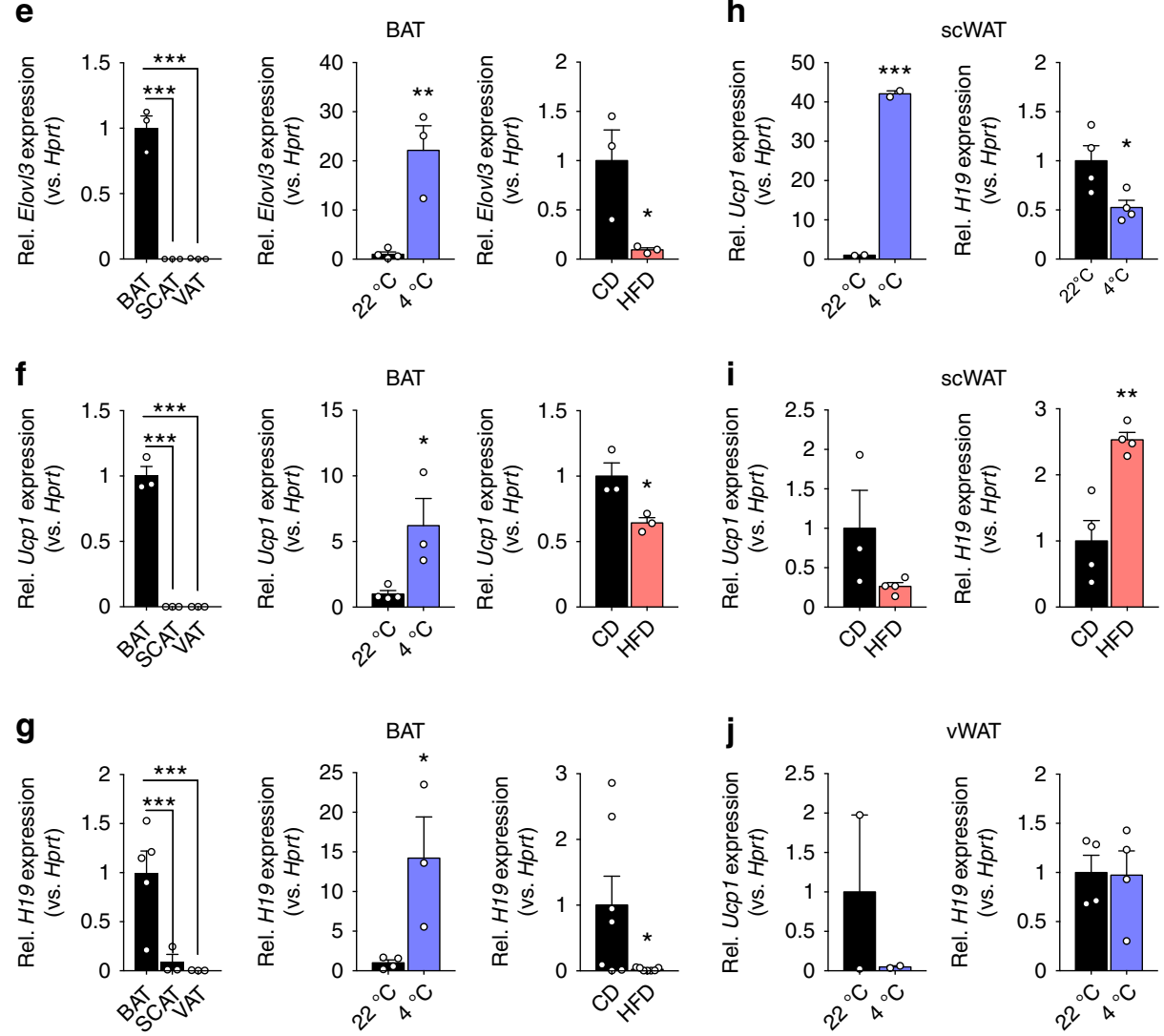
miR-675 in brown adipocyte differentiation as observed for myogenic lineage determination ${ }^{23}$.

To gain insights into $H 19$ function from global expression analysis, we performed RNA-Seq in differentiating siRNA-treated brown adipocytes, and identified 1410 differentially expressed genes $(p<0.05$, DESeq2), comprising 546 decreased and 999 enriched in siH19 versus siCtrl cells (Fig. 2j). Lower-expressed genes were linked to brown adipocyte and mitochondrial biogenesis and function, which failed to be expressed upon H19 silencing (Fig. 2j, k top) and Supplementary Data 3), whereas higher-expressed genes were enriched for general functions in signaling, locomotion, and morphogenesis, processes normally suppressed during adipogenesis ${ }^{24-26}$ (Fig. $2 \mathrm{j}$, k bottom and Supplementary Data 3). Gene set enrichment analysis (GSEA) ${ }^{27}$ indicated significant enrichment of gene sets for brown adipogenesis, oxidative phosphorylation (OxPhos), and mitochondrial biogenesis specifically in cells with intact H19 expression (siCtrl; Fig. 2l). Suppression of the brown adipogenesis program upon H19 knockdown could be due to suppression of genes important for adipogenesis in general. To test this, we investigated the consequences of H19 RNAi on expression of previously defined groups of BAT-specific, WAT-specific, and common adipogenic genes in differentiated BAT precursor cells $^{25}$. We found that, in general, loss of H19 repressed BATselective as well as those common adipogenic genes normally activated during adipogenesis (Fig. $2 \mathrm{~m}$ ), which is also reflected by reduced ORO staining (Fig. 2f). In contrast, WAT-selective genes are modestly affected by $H 19$ knockdown (Fig. 2m, n). Collectively, $\mathrm{H} 19$ mediates the concurrent activation of common adipogenic genes as well as a core BAT gene program, whilst curbing the expression of WAT genes.

H19 increases BAT EE and prevents obesity. Defects in brown fat differentiation and function as well as concomitant impairments in EE render mice susceptible to DIO-induced weight gains and the development of metabolic disease ${ }^{28-30}$. Based on our in vitro findings, we next addressed the role of $\mathrm{H} 19$ in regulating energy metabolism and EE by exposing $H 19$ gain-of-function ${ }^{31}$ and Controls to HFD or CD. H19 expression was ubiquitously induced in H19 TG mice without changes in Igf2 and Ctcf expression or body length alterations (Supplementary Fig. 3a-d). H19 overexpression strongly prevented DIO-mediated weight gains (Fig. 3a) and improved insulin sensitivity (Fig. 3b) although, interestingly, glucose tolerance was decreased in H19 TG mice (Fig. 3c). The beneficial effect of H19 overexpression in DIO coincided with increased EE (Fig. 3d), trends towards increased lipid mobilization at $22^{\circ} \mathrm{C}$ (Fig. 3e) and oxygen consumption (Supplementary Fig. $3 \mathrm{e}$ ) at $22^{\circ} \mathrm{C}, 4^{\circ} \mathrm{C}$ and after additional norephinephrine (NE) administration at $4{ }^{\circ} \mathrm{C}$, pinpointing towards improved BAT function in H19 TG mice. This rise in energy catabolism tended towards prevention of DIO-evoked increases in scWAT and vWAT adiposity (Fig. 3f) and precluded DIOassociated elevations in serum cholesterole (Fig. $3 \mathrm{~g}$ ) but not triglycerides (Fig. 3h). H19 overexpression prevented whitening of BAT (Fig. 3i, j), reduced vWAT but not scWAT hypertrophy (Fig. 3k-m) and development of steatosis (Fig. 3n) in obese animals. The H19-dependent induction of EE in obese mice cooccurred with increased expression of browning markers in scWAT at $4{ }^{\circ} \mathrm{C}$, with little alterations in BAT thermogenesis at this temperature (Fig. 3o, p) as reported by others ${ }^{29}$. As H19 overexpression in lean mice elicited little changes in body weight, insulin sensitivity, RER, glucose clearance, EE and did not affect relative adipose tissue weights and expression of BAT activation markers in lean H19 TG mice (Supplementary Fig. 3f-k), our data suggests that counteracting the HFD-induced H19 decline is beneficial during DIO, but does not cause detrimental effects in lean mice.

$\mathrm{H} 19$ loss in fat impairs EE and sensitizes towards obesity. As H19 TG mice ubiquitously overexpress H19, we next ablated H19 specifically in BAT by crossing female mice harboring loxPflanked $H 19$ methylation-sensitive imprinting control regions (ICRs) with male Adipoq-cre expressing animals (H19 ${ }^{\Delta \mathrm{AT}}$, Fig. 4a, Supplementary Fig. 4a, b). H19 ${ }^{\Delta \mathrm{AT}}$ knockout mice gained more body weight compared to Controls upon HFD (Fig. 4b) but not CD (Supplementary Fig. 4c) feeding with negligible alterations in insulin sensitivity or glucose tolerance (Fig. 4c, d and Supplementary Fig. 4d, e). Crucially, BAT-specific H19 loss decreased EE and oxygen consumption in both diets (Fig. 4e, $\mathrm{f}$ and Supplementary Fig. 4f, g), tended towards decreased lipid oxidation at $4{ }^{\circ} \mathrm{C}$ and increased scWAT adiposity in obese (Fig. 4g, h), yet not lean (Supplementary Fig. 4h, i) mutant mice, suggesting decreased BAT function in $\mathrm{H} 19^{\Delta \mathrm{AT}}$ mice, particularly during DIO. As primary brown adipocytes isolated from $\mathrm{H} 19^{\Delta \mathrm{AT}}$ mice exhibited defects in mature brown adipocyte function (Supplementary Fig. 4j, k) as partly also observed during H19 RNAi (Supplementary Fig. 4p), we reasoned that impairments in terminal brown function contribute to the observed defects in $\mathrm{EE}$ and susceptibilities towards DIO in H19 $\mathrm{AAT}$ mice.

Fat hH19 is reduced in obese humans. As $H 19$ is strongly conserved $^{32}$ and human $H 19(h H 19)$ is expressed in mature adipocytes compared to non-parenchymal cells in fat (Supplementary Data 4), we next asked if $h H 19$ could be affected by obesity also in humans and quantified $h H 19$ levels in scWAT and vWAT biopsies from 169 lean and obese patients. We observed that $h \mathrm{H} 19$ expression declined with ascending BMIs in both depots and correlated positively with markers of adipose beiging like UCP1 mRNA levels (Supplementary data 5), underscoring that $h H 19$ could support energy dissipation and acquisition of catabolic expression profiles in human fat.

H19 regulates brown adipose mitochondrial biogenesis. Cold temperature activation of BAT thermogenesis and the concurrent turnover of nutrients, such as carbohydrates and lipids requires active mitochondrial biogenesis and rearrangements of

Fig. 1 RNA-Seq reveals lincRNAs correlating with brown fat function in vivo. a, b Plot of BAT (a) mRNA or (b) lincRNA expression after HFD or CD feeding (RNA-Seq, $n=2$ per biological condition). c, d Plot of BAT c mRNA or $\mathbf{d}$ lincRNA expression after $24 \mathrm{~h}$ of $4{ }^{\circ} \mathrm{C}$ or $22^{\circ} \mathrm{C}$ (RNA-Seq, $n=2$ per biological condition). e-g BAT, scWAT, and VWAT expression of e Elov/3 $\mathbf{f} U \mathrm{cp} 1$, and $\mathbf{g} H 19$ expression in CD-fed mice ( $n=3-5$ per given tissue, left), in CD-fed mice housed at $22^{\circ} \mathrm{C}(n=4)$ versus $24 \mathrm{~h}$ of $4{ }^{\circ} \mathrm{C}(n=3$, middle) and HFD-fed $(n=3$ for Elovl3 and Ucp1, $n=7$ for H19) versus CD-fed mice ( $n=3$ for Elov/3 and $\mathrm{Ucp} 1, n=7$ for H19, right, qRT-PCR. h, i scWAT expression of Ucp1 and $\mathrm{H} 19$ in $\mathbf{h}$ mice housed at $22^{\circ} \mathrm{C}(n=2$ for Ucp $1, n=4$ for $H 19)$ versus $24 \mathrm{~h}$ of $4{ }^{\circ} \mathrm{C}$ ( $n=2$ for Ucp1, $n=4$ for H19) and $\mathbf{i}$ HFD-fed $(n=4)$ versus CD-fed ( $n=3$ for Ucp1, $n=4$ for H19) mice. $\mathbf{j}$ vWAT Ucp1 and H19 expression in mice housed at $22^{\circ} \mathrm{C}$ versus $24 \mathrm{~h}$ of $4{ }^{\circ} \mathrm{C}(n=2$ for $U c p 1, n=4$ for H19). Unless stated, bar graphs represent mean \pm s.e.m. with all data points plotted and unpaired, two-tailed Student's $t$-tests were used to assess statistical significance. ${ }^{\star} p<0.05$, ${ }^{\star \star} p<0.01,{ }^{\star \star \star} p<0.001$. If applicable $p$-values are indicated within the panel 
mitochondrial meshworks via fission and fusion ${ }^{33,34}$. Based on the cell-intrinsic alterations in oxidative metabolism observed in H19-deficient and overexpressing adipocytes (Fig. 2d, e, Supplementary Fig. 1a, b) we studied mitochondrial morphologies using electron microscopy. Although gross parameters like mitochondrial area or perimeter (Fig. $4 \mathrm{i}, \mathrm{j}$ ) were unchanged, we observed substantial defects in mitochondrial architecture such as perturbed cristae formation in obese Control but not H19 TG mice (Fig. 4k), while $\mathrm{H} 19^{\triangle \mathrm{AT}}$ mitochondria showed impaired cristae upon HFD feeding (Supplementary Fig. 4q). The expression of genes involved in mitochondrial biogenesis was blunted in obese BAT, whilst H19 overexpression reversed these DIOevoked defects in gene expression in BAT, but not scWAT (Fig. 4l, m). H19 ${ }^{\Delta \mathrm{AT}}$ mice displayed less pronounced reductions a
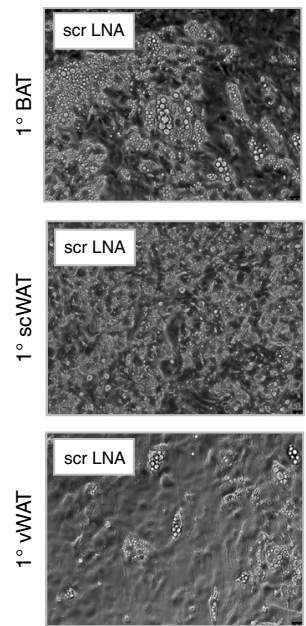

b
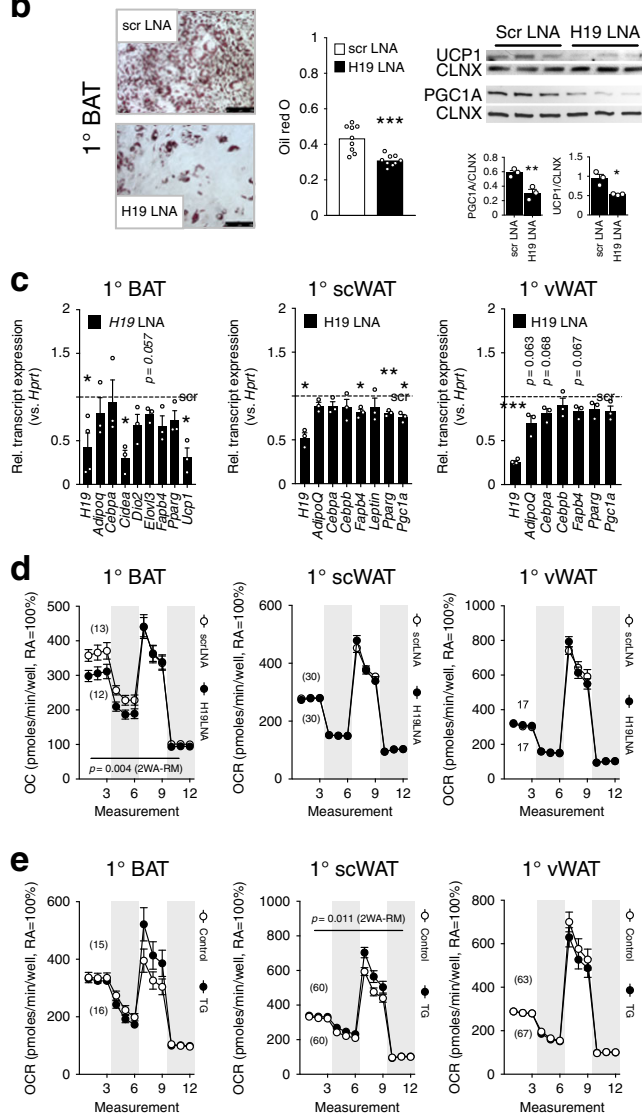

$\mathbf{f}$

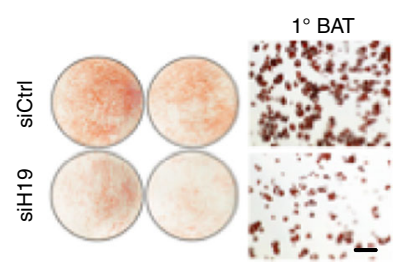

g
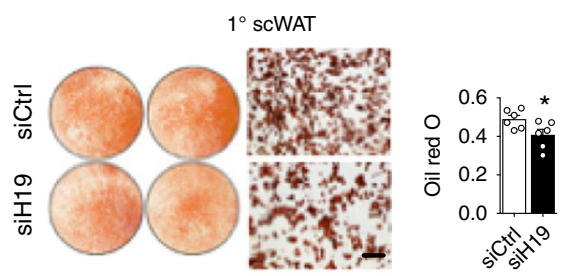

h
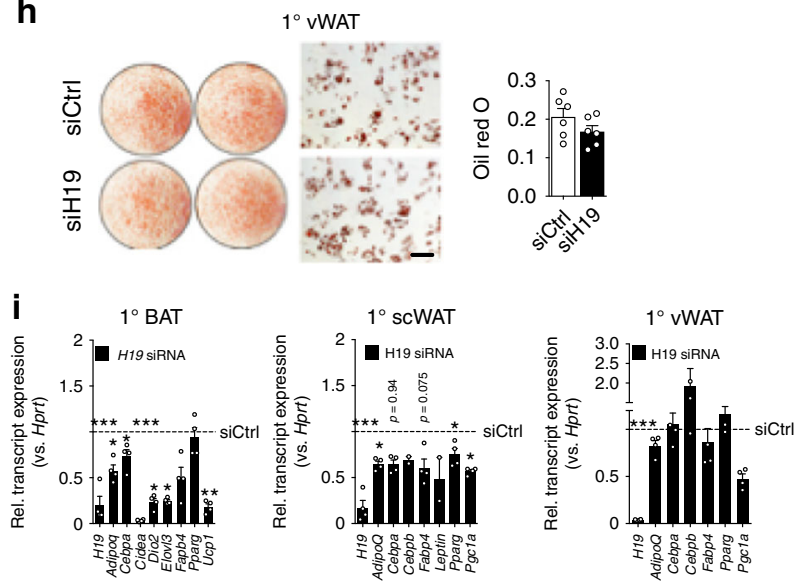

k
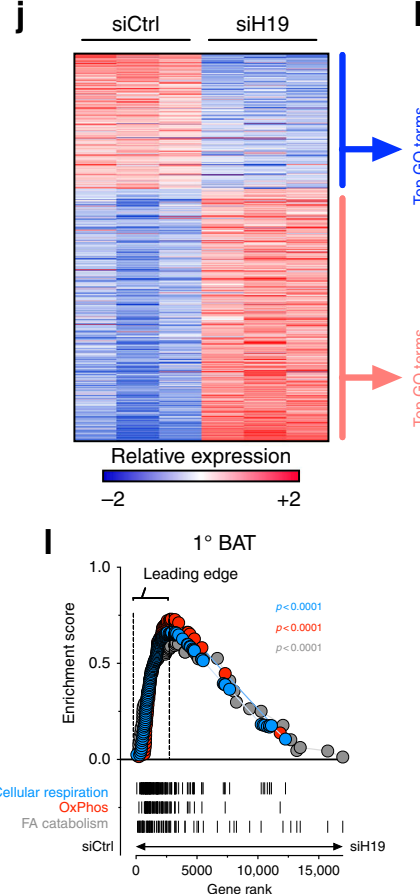

m
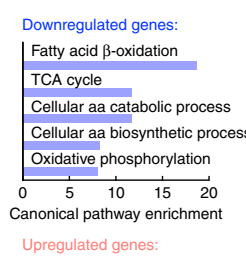

Muscle contraction

RPTK signaling

Locomotion

Cellular component morphogenesis Anatomical structure morphogenesis

\begin{tabular}{lllll}
\hline & 1 & 1 & 1 & 1 \\
0 & 5 & 10 & 15 & 20
\end{tabular}

m $\quad 1^{\circ} \mathrm{BAT}$

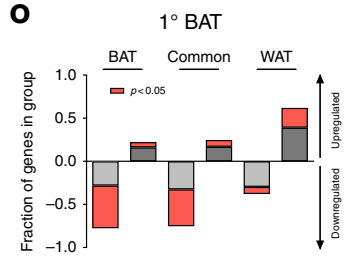

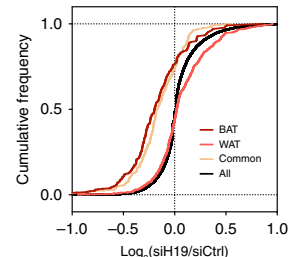

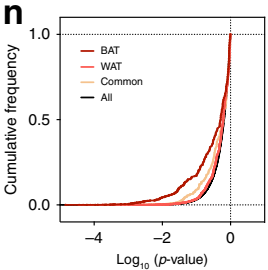


of the same gene set in BAT (Fig. 4n) yet not scWAT (Fig. 4o). In contrast, fission and fusion parameters were unaltered in BAT and scWAT (Supplementary Fig. 4l-o). Interestingly, cellintrinsic defects in mitochondriogenesis occurred also in H19deficient brown adipocytes (Supplementary Fig. 4p), closely mimicking those elicited by DIO or H19 loss in adipose tissue itself. Collectively, we here show that $H 19$ loss in adipose tissue renders mice susceptible to DIO weight gains and impairments of $\mathrm{EE}$, potentially due to the control of mitochondrial dynamics by the lincRNA $H 19$.

BAT represents a unique case of tissue-specific imprinting. The lincRNA $H 19$ represents a quintessential representative of a class of imprinted genes exclusively transcribed from one parental allele 20,35 . Although monoallelic expression is only observed in $<1 \%$ of all genes in eutherians ${ }^{36}$, loss-of-function (epi)-mutations in individual imprints or imprinted gene clusters cause severe imprinting disorders (ID) like Prader-Willi's, Beckwith-Wiedemann's, and Angelman's syndromes ${ }^{37}$, in Silver-Russell's syndrome even due to defects in the H19-Igf2 locus $^{38}$. Despite progress in understanding the molecular underpinnings of imprinting, the etiology of IDs $s^{21,39,40}$ and even recent approaches to correct $\mathrm{IDs}^{41}$, the question remains why certain genes but not others are monoallelically expressed per $\mathrm{se}^{42}$ and why certain genes are expressed from one parental gender, not the other. Thus, to date it remains unsolved whether functional or conceptual similarities between paternally expressed (PEGs) or maternally expressed genes (MEGs) as two discordant gene sets exist at all, largely because PEGs/MEGs represent a collection of genes with heterogeneous functions. Interestingly, one hallmark of ID patients and ID mouse models are metabolic problems, for instance altered adiposity and lipodystrophy and alterations in insulin sensitivity and $\mathrm{EE}^{43}$, all processes closely linked to brown and WAT (dys)-function. Given the BATselectivity of $\mathrm{H} 19$ and its novel role in brown adipocyte differentiation and function, we were interested how other PEGs/ MEGs are expressed across fat depots transcriptome-wide. While RNA-Seq from C57BL/6 mice revealed no gross differences in MEGs abundances between BAT, scWAT, or vWAT, seven fatabundant protein-coding (G Protein Coupled Receptor 1 (Gpr1), Insulin Like Growth factor 2 (Igf2), Mesoderm Specific Transcript (Mest), Neuronatin (Nnat), Paternally Expressed 3 and 10 (Peg3, Peg10), and Plag1 Like Zinc Finger 1 (Plagl1)) and three microRNA PEGs ( $m i R 184, m i R 298$, and $m i R-335$, Fig. 5e) and many others were absent from BAT yet robustly expressed in scWAT and vWAT (Fig. 5a-e).
H19 curbs expression of PEG-enriched gene networks in BAT. To shed molecular light on this unique pattern of fat tissuespecific imprinting, we built upon recent reports demonstrating that expression of imprinted genes can be interdependent, e.g., during muscle regeneration or in embryonic fibroblasts ${ }^{44-47}$ and that PEG-enriched imprinted gene networks (IGNs) are affected by $H 19^{48-50}$. We thus hypothesized that $H 19$ represses PEG-IGNs in brown fat and, indeed, H19 RNAi tended to derepress fatabundant PEGs like Igf2, Peg10, and Plagl1 but not MEGs like Cyclin Dependent Kinsae Inhibitor 1C (Cdkn1c), Decorin (Dcn), Igf2 Receptor (Igf2r), and Solute Carrier Family 22 Member 3 (Slc22a3) (Fig. 5f, g). As PEG expression declined brown adipogenesis (Fig. 5h), we next asked if H19 ablation affects PEG/ MEGs globally. As expected, $H 19$ loss did not alter MEG expression in differentiated primary $\left(1^{\circ}\right)$ BAT or $1^{\circ} \mathrm{vWAT}$ precursor cells (Supplementary Fig. 5a, b), yet counteracted differentiation-induced PEG losses during brown, but not white adipogenesis (Fig. 5i, j). Thus, H19 acts as PEG gatekeeper in brown adipocytes, a finding not observed for BAT MEGs nor white fat PEGs.

H19 represses BAT PEGs by recruiting MBD1 chromatin modifier. As H19 expression was not altered in BAT, scWAT, and vWAT commitment (Supplementary Fig. 5c) and because we did not observe differences in DNA methylation of affected PEGs ICRs (Supplementary Fig. 5d), we asked whether specific protein interactors of $\mathrm{H} 19$ control its PEG gatekeeper function selectively in brown adipocytes. We thus conducted Capture Hybridization Analysis of RNA Targets ${ }^{51}$ coupled to mass spectrometry (CHART-MS) from ${ }^{-}$confluent and differentiated primary immortalized brown adipocytes (PIBA) and obtained a comprehensive $H 19$ protein interactome from both conditions: In total 3590 proteins were detected by MS, 168 were enriched by H19 antisense, not sense oligonucleotide co-immunoprecipitation (Supplementary Data 7). 76 and 54 proteins bound to H19 in confluent and differentiated PIBA cells, respectively, and 38 proteins were bound by $\mathrm{H} 19$ in both (Fig. 5k). Next, we performed Ingenuity Pathway Analysis to construct an interconnected $\mathrm{H} 19$ binding protein network comprising 61 proteins (Fig. 5l). Crucially, when performing gene ontology (GO) analyses, we observed that whereas in immature (confluent) brown adipocytes $H 19$ mostly recruited proteins annotated as RNAbinding proteins (RBPs, e.g., H19 interactors like Insulin Like Growth Factor 2 Binding Protein 3 (IGFBP3) $)^{52}$, in differentiated cells $H 19$ preferentially associated with proteins classified as

Fig. 2 LincRNA H19 required for brown but not white adipocyte differentiation and function. a Photomicrograph of BAT, scWAT, and vWAT $1^{\circ}$ adipocytes transfected with scrambled (scr) or H19 LNAs. Pictures represent $n=6\left(1^{\circ} \mathrm{BAT}\right)$ or $n=3$ (others) experiments, $n=3$ replicates each. Scale bar, $100 \mu \mathrm{m}$ b ORO staining and quantification, PGC1A/UCP1 immunoblot and quantification of 1 ${ }^{\circ} \mathrm{BAT}$ transfected with scr or H19 LNA. Scale bar, 250 um. c Expression of indicated mRNAs in BAT, scWAT and vWAT $1^{\circ}$ adipocytes transfected with scr or H19 LNAs. A paired, two-tailed Student's $t$-test was used to assess significance across $n=3$ experiments, $n=3$ replicates each. d Oxygen consumption rates (OCR) in $1^{\circ}$ adipocytes from indicated depots transfected with scr or H19 LNA. Alternating backgrounds depict medium, oligomycin, FCCP, and rotenon plus antimycin A injections. Numbers of measured wells are indicated in brackets. e OCRs in $1^{\circ}$ adipocytes from indicated depots from $\mathrm{H} 19$ TG or Controls. d, e A two-way ANOVA with repeated measurements (2WA-RM) was applied to assess significance. $\mathbf{f}-\mathbf{h}$ ORO-stained 6-well plates, ORO photomicrographs and ORO densitometry in $\mathbf{f}$ BAT, $\mathbf{g}$ scWAT, and h vWAT $1^{\circ}$ adipocytes transfected with $H 19$ (siH19) versus control (siCtrl) siRNAs. An unpaired, two-tailed Student's t-test was applied to assess significance. Scale bar, $250 \mu \mathrm{m}$ i Expression of indicated mRNAs transfected with siH19 or siCtrl. An unpaired, two-tailed Student's $t$-test was applied to assess significance across $n=3-4$ experiments, $n=3$ replicates each. $\mathbf{j}$ Expression change of 1410 mRNAs differentially expressed ( $p<0.05$, fc $\geq 1.3-f o l d$ ) in siH19-transfected mature brown adipocytes. k Top $5 \mathrm{GO}$ terms enriched ( $p<0.05$, Bonferroni correction) among mRNAs showing significantly higher (top) or lower (bottom) expression $(p<0.05$ ) upon siH19 versus siCtrl. I GSEA for brown adipogenesis, OxPhos, and mitochondrial biogenesis. $p$ empirical $p$-value. $\mathbf{m}, \mathbf{n}$ Cumulative distributions of expression changes $(\mathbf{m})$ and p-values $(\mathbf{n})$ for all, BAT-specific, WAT-specific, and common adipogenic genes in siH19-treated mature brown adipocytes. $\mathbf{j}, \mathbf{m}, \mathbf{n}$ Changes are siH19 log2 expression (FPKM) ratios over siCtrl. o Proportion of gene sets upregulated $(\mathrm{L} 2 \mathrm{R}>0)$ or downregulated $(\mathrm{L} 2 \mathrm{R}<0)$ in siH19-treated cultured mature brown adipocytes. Unless stated, bar graphs represent mean \pm s.e.m. with all data points plotted for $\left(\mathbf{b}, \mathbf{c}, \mathbf{f}-\mathbf{h}, \mathbf{i} .{ }^{\star} p<0.05,{ }^{\star \star} p<0.01,{ }^{\star \star \star} p<0.001\right.$. If applicable $p$-values are indicated 
chromatin binders or chromatin modifiers (Fig. 5l, m). Of note, H19 bound DNA methyltransferase MBD1 (and its homolog MECP2) only in differentiated PIBA cells. Crucially, H19-MBD1 complexes affect histone 3 lysine methyltransferases (KMT) dependent deposition of repressive $\mathrm{H} 3 \mathrm{~K} 27$ trimethylation marks and $\mathrm{H} 19$ or MBD1 loss both resulted in reduced H3K9me3 at PEG loci like Igf2, Solute Carrier Family 38 Member 4 (Slc38a4) and Mest/Paternally Expressed 1 (Peg1 $)^{50}$. Accordingly, we observed in primary mature brown adipocytes that siH19 and/or siMbd1 RNAi increased BAT-abundant PEGs with similar tendencies, arguing for functional H19/MBD1 complexes in these cells (Supplementary Fig. 5e). Presence of H19-MBD1 complexes occurring solely in mature brown adipocytes thus supports the notion of $H 19$ as PEG gatekeeper in brown adipocytes, potentially via MBD1-dependent alterations of H3K9me3 KMTs recruitment. Crucially, the same phenomenon a
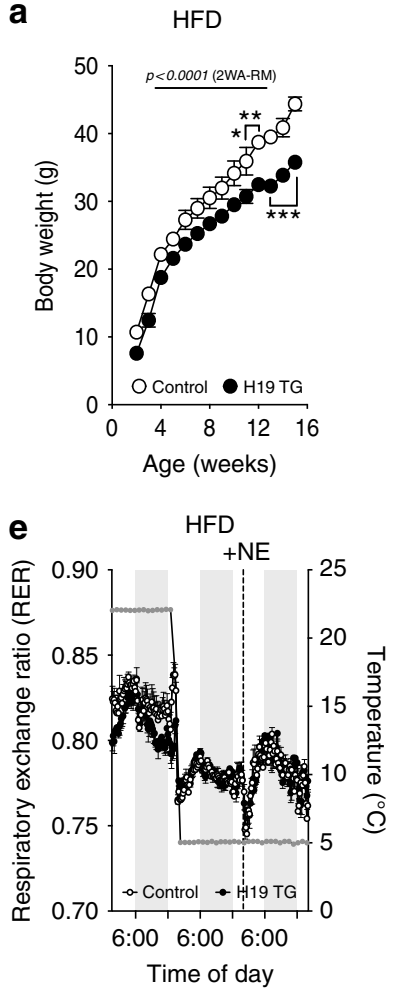

i
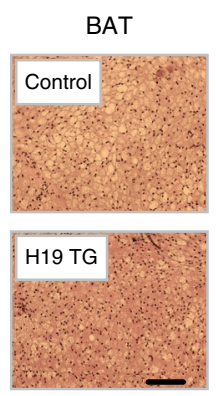

m

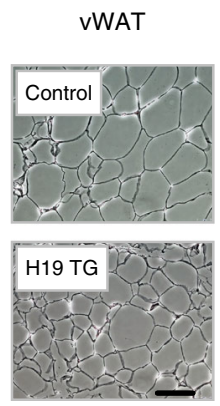

b

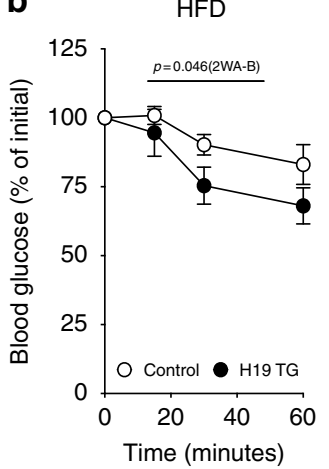

f

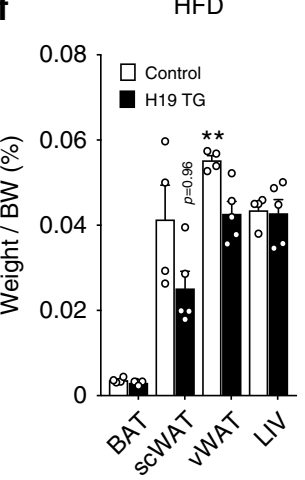

c

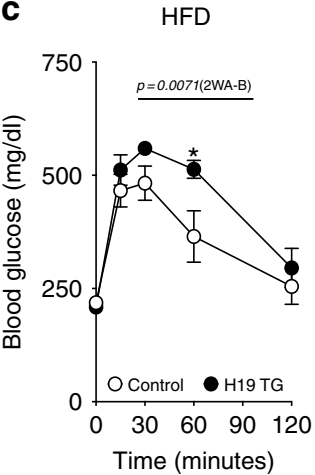

g

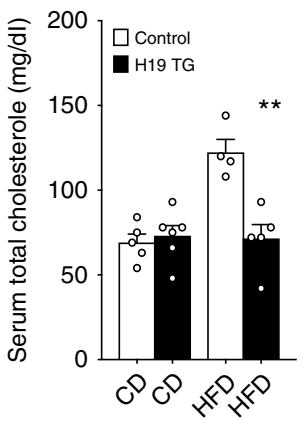

k

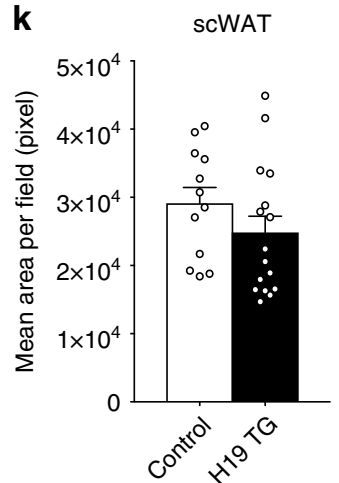

n
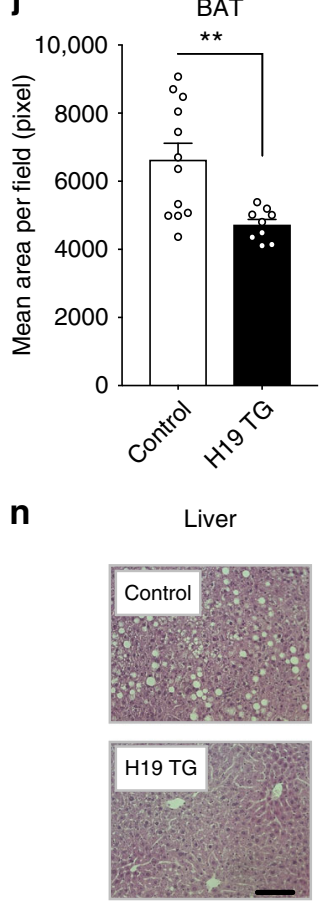

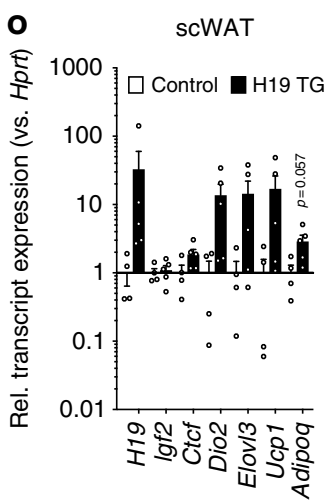

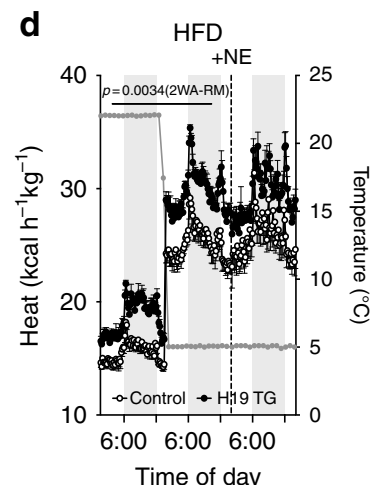

h
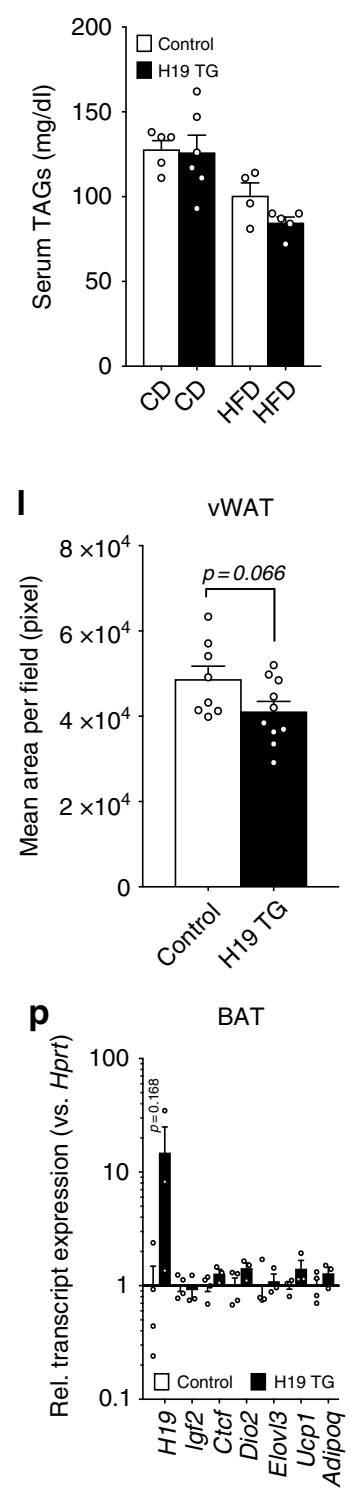
does not occur in white adipocytes where H19 abundances are low.

We reasoned that PEG enrichment in subcutaneous and visceral WAT pinpoints towards an underappreciated involvement of PEGs in WAT-relevant processes like white adipogenesis or lipid accrual, i.e.processes that sensitize towards increased adiposity and obesity. To address this, we quantified PEGs/ MEGs in DIO using RNA-Seq and, performing GSEA ${ }^{27}$, observed trends towards decreased PEG levels in cold-exposed (Fig. 5n) and elevated PEG expression in obese BAT (Fig. 5o). In an elegant study, Koza et al. biopsied adipose tissue from C57BL6 mice before HFD feeding ${ }^{53}$. Profiling the pretreatment samples from low and high BW gainers of the 107-animal cohort, the authors reported gene signatures for DIO susceptibility. Reanalyzing this data set, we found clear evidence that PEG levels foreshadow obesity susceptibility even before overnutrition (Fig. 5p). Finally, H19 correlated negatively, whereas 5 of 10 fat-abundant PEGs (Gpr1, Mest, Nnat, Peg3, and Sgce) correlated positively with DIO susceptibility across a panel of 25 phenotypically and genetically discordant mouse strains ${ }^{54}$ (Fig. 5p-r, Supplementary Fig. 5f). Taken together, we believe that H19-MBD1 complexes repress PEG but not MEG expression in brown adipocytes, supporting a notion according to which individual PEGs ${ }^{47,55,56}$ but also paternal monoallelic gene expression in general sensitizes towards fat accumulation and diet-induced weight gains, a process at least partially repressed by the lincRNA H19 in BAT.

Collectively, we here demonstrate that the conserved lincRNA $\mathrm{H} 19$ is uniquely required for differentiation and mature fat cell function of brown but not white adipocytes in vitro and that brown adipose $H 19$ ensures energy dissipation in vivo. One mechanism of how $\mathrm{H} 19$ supports EE could be its cell-intrinsic control of mitochondrial biogenesis in mature brown adipocytes and the ensurance of maintaining quiescence of obesitypredisposing PEGs in BAT.

\section{Discussion}

Our results collectively indicate that the conserved lincRNA H19 promotes brown adipocyte differentiation and function in vitro and that $H 19$ ensures energy dissipation in vivo. In addition, we observed that H19-mediated alterations of IGN constitutes an important regulatory layer of BAT development and function according to which PEGs affect brown fat negatively (Dlk1, Peg1, $N d n$, paternally expressed GNAS isoform XLas), whereas MEGs (Gas) affect BAT positively ${ }^{43}$. The kinship theory of the evolution of imprinting predicts that PEGs should act to reduce energy- costly thermogenic output ${ }^{57}$. Our data are broadly consistent with this prediction as loss of $\mathrm{H} 19$ in brown adipocytes leads to coordinated transcriptional upregulation of multiple paternally imprinted genes. This finding is distinct from canonical imprinting regulation in that perturbed imprinting control should lead to downregulation of some imprinted genes with a reciprocal upregulation of others.

We obtained no evidence for changes in canonical imprinting in our models, amongst others because DNA methylation of affected PEGs ICRs and Igf2/Ctcf expression was unaltered by H19 loss in brown adipocytes. Our data rather suggest that this unique pattern of PEG regulation is maintained through $\mathrm{H19} /$ Mbd1 complexes acting in trans. Although evidence supporting this was collected from a single Mbd1 siRNA, MBD1 was already shown to interact with $\mathrm{H} 19$ to regulate IGNs during embryo growth ${ }^{50}$. Yet, future studies are needed to show if H19 PEG gatekeeper function persists at thermoneutrality or other (e.g., surgical) models of altered BAT-evoked EEs.

Because of the role of PEGs in WAT-relevant processes like white adipogenesis and adipocyte cell size regulation, it is plausible to assume that H19 preserves BAT homeostasis during DIO by its cell-intrinsic control of mitochondrial biogenesis in mature brown adipocytes and by maintaining quiescence of obesity-predisposing PEGs in BAT. In line with this hypothesis are data showing brown adipocyte death as a consequence of DIO induce whitening of $\mathrm{BAT}^{58}$, a process suppressed by $\mathrm{H} 19$.

Collectively, our data suggest that $H 19$ acts as PEG gatekeeper in brown adipocytes, potentially acting via MBD1-dependent alterations of $\mathrm{H} 3 \mathrm{~K} 9 \mathrm{me} 3 \mathrm{KMTs}$ recruitment are in line with previous reports demonstating that $\mathrm{H} 19$ regulates fine-tuned regulation of embryonic growth mediated by the H19 gene. Identification of the underlying molecular mechanisms through which it controls its targets is an important issue.

\footnotetext{
Methods

Animal care and research diets. All animals were maintained on a C57BL/6 background, housed in groups of 3-5 animals per cage on a constant $12 \mathrm{~h}$ light/ dark cycle in a SPF-controlled facility with regular testing for pathogens. All experimental mice were 17-18 weeks of age at sacrifice. Experimental mice were exposed to HFD feeding for 15 weeks (H19 TG and H19 ${ }^{\Delta \mathrm{AT}}$ mice versus littermate Controls) and 17-18 weeks of age at sacrifice. Care of animals was within institutional and animal-care committee guidelines approved by local (Bezirksregierung Köln) or regional (Tierschutzkommission acc. \$15 TSchG of the Landesamt for Natur, Umwelt und Verbraucherschutz (LANUV) North-Rhine Westphalia, Germany, internal accession no. 84-02.04-2014.A068) authorities. Unless otherwise indicated, animals were allowed ad libitum access to control diet $\left(C D, D 12450 B^{\star}\right.$ mod LS; Sniff) containing $62 \mathrm{~kJ} \%$ carbohydrates, $27 \mathrm{~kJ} \%$ protein, and $11 \mathrm{~kJ} \%$ fat and drinking water. DIO was achieved by feeding a high-fat diet (HFD, D12492 (I)
}

Fig. $3 \mathrm{H} 19$ overexpression protects from obesity by increasing energy expenditure and scWAT beiging. a Body weight of HFD-fed Control ( $n=4-5)$ versus H19 TG ( $n=4-5)$ mice. A 2WA-RM with Bonferroni post hoc correction for multiple comparisons (2WA-B) was applied to assess significance. $\mathbf{b}$ Insulin tolerance test of HFD-fed Control $(n=4)$ versus H19 TG $(n=6)$ mice. c Glucose tolerance test of HFD-fed Control $(n=5)$ versus H19 TG $(n=5)$ mice. d Energy expenditure in HFD-fed Control $(n=4)$ versus H19 TG $(n=5)$ mice. b-d A 2WA-B was applied to assess significance. e Respiratory Exchange Ratios (RER) in HFD-fed Control $(n=4)$ versus H19 TG $(n=5)$ mice. $\mathbf{f}$ Tissue/body weight (BW) ratios in HFD-fed Control $(n=4)$ versus H19 TG $(n=5)$ mice. An unpaired, two-tailed Student's t-test was applied to assess significance. $\mathbf{g}$ Serum cholesterole levels in HFD-fed Control ( $n=4$ ) versus H19 TG $(n=5)$ mice. $\mathbf{h}$ Serum triglyceride levels in HFD-fed Control $(n=4)$ versus H19 TG $(n=5)$ mice. An unpaired, two-tailed Student's $t$-test was applied to assess significance. i Representative photomicrograph of BAT from HFD-fed Control versus H19 TG mice. $\mathbf{j}$ Automated quantification of adipocyte mean area per field in hematoxylin/eosin (HE) stained BAT sections from HFD-fed Control ( $n=12$ fields) versus H19 TG ( $n=9$ fields). An unpaired, two-tailed Student's $t$-test was applied to assess significance. $\mathbf{k}$ Automated quantification of adipocyte mean area in HE-stained scWAT sections from HFD-fed Control ( $n=12$ fields) versus H19 TG ( $n=16$ fields) mice. I Automated quantification of adipocyte mean area in HE-stained vWAT sections from HFD-fed Control ( $n=8$ fields) versus H19 TG ( $n=10$ fields) mice. An unpaired, two-tailed Student's $t$-test was applied to assess significance. $\mathbf{m}$ Representative photomicrograph of vWAT sections from HFD-fed Control versus H19 TG mice. $\mathbf{n}$ Representative photomicrograph of liver sections from HFD-fed Control versus H19 TG mice. $\mathbf{m}, \mathbf{n}$ Scale bar, $50 \mu \mathrm{m}$. o scWAT expression of indicated mRNAs from HFD-fed Control $(n=4)$ versus H19 TG $(n=5)$ mice. $\mathbf{p}$ Relative BAT expression of indicated mRNAs from HFD-fed Control $(n=4)$ versus H19 TG mice $(n=3)$. An unpaired, two-tailed Student's $t$-test was used to assess significance in $\mathbf{0}, \mathbf{p} .{ }^{\star} p<0.05,{ }^{\star \star} p<0.01,{ }^{\star \star \star} p<0.001$. If applicable $p$-values are indicated within the panel 
mod; Sniff) containing $22 \mathrm{~kJ} \%$ carbohydrates, $24 \mathrm{k} \mathrm{J} \%$ protein, and $54 \mathrm{~kJ} \%$ fat from starting at 3 weeks of age.

Mouse husbandry. Adipose tissue samples used for acquisition of a tissue RNASeq profile were taken from male C57BL/6 mice (Charles River). At time of sacrifice, the mice were 32 weeks of age and 28 weeks exposed to CD feeding. C57BL/6 male mice used for CD versus HFD feeding came from Charles River. Upon dissection, the mice were 32 weeks of age and 28 weeks exposed to CD or HFD feeding. For cold exposures, 10-12 weeks old C57BL/6 male mice were either constantly housed at $22^{\circ} \mathrm{C}$ or exposed to $4{ }^{\circ} \mathrm{C}$ cold challenges for $20 \mathrm{~h}$, fasted for additional $4 \mathrm{~h}$, and sacrificed thereafter. $\mathrm{H} 19$ overexpression was achieved using a mouse strain harboring yeast artificial chromosomes (YACs, present in ten genomic copies) with each YAC cassette containing a $130 \mathrm{~kb}$ transgene spanning the entire H19-Igf2 cluster with a LacZ-inactivated Igf2 coding sequence (MGI accession number 5648556, Symbol: Tg(Igf2/LacZ,H19) YZ15Aco; H19 TG) ${ }^{31}$. Non-transgenic littermates were used as controls. Only male, non-randomized mice were used, the investigator not blinded to genotypes due to strong phenotypical differences (e.g., in body weight upon HFD feeding) between H19 TG and Controls. Mice harboring loxP-flanked H19 differentially methylated regions (DMR; H19-DMR ${ }^{\text {flDMR/flDMR }}$ ) were generated as described ${ }^{59}$ and obtained from Linheng Li (Stowers Institute). a
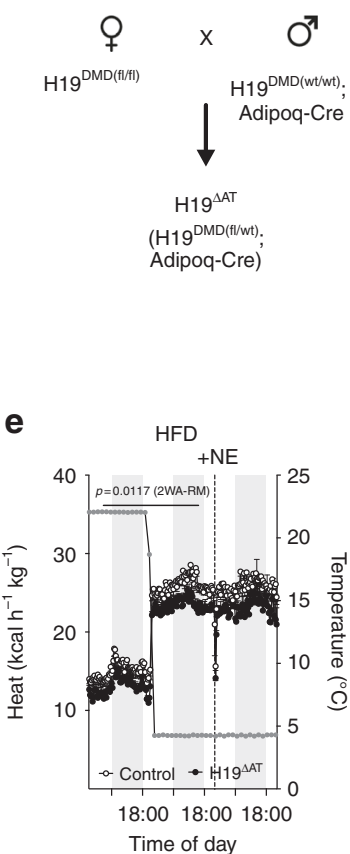

i

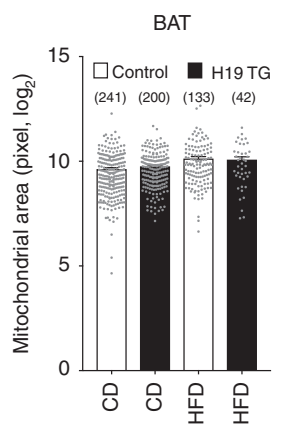

I

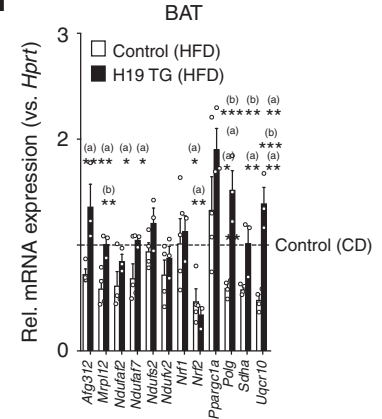

Mitochondrial biogenesis b

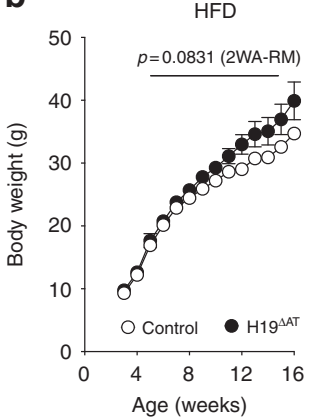

f

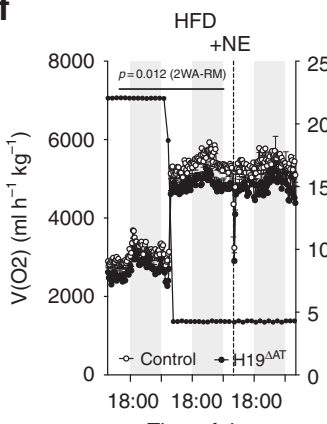

Time of day

j

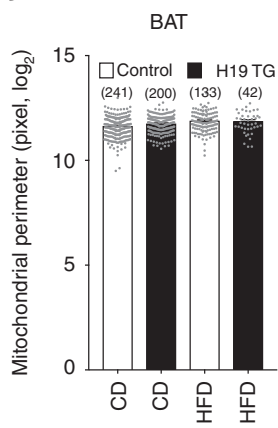

m

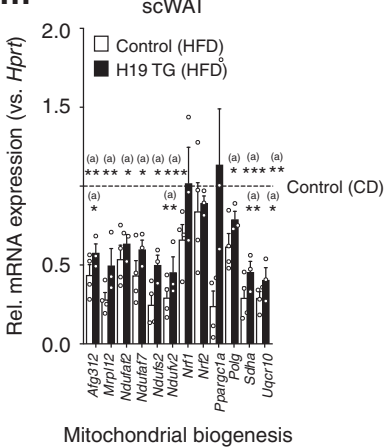

C

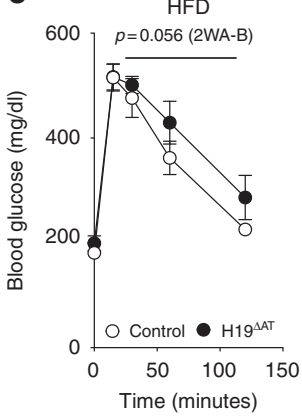

g
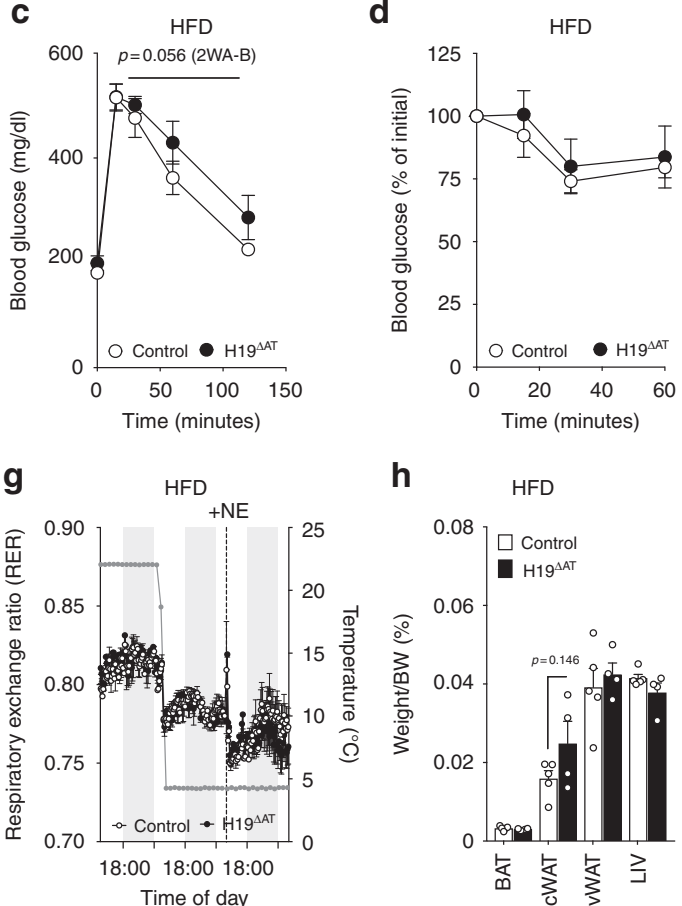

h

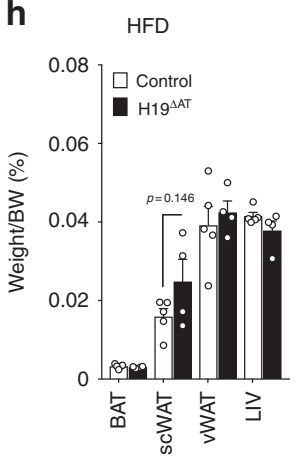

$\mathbf{k}$
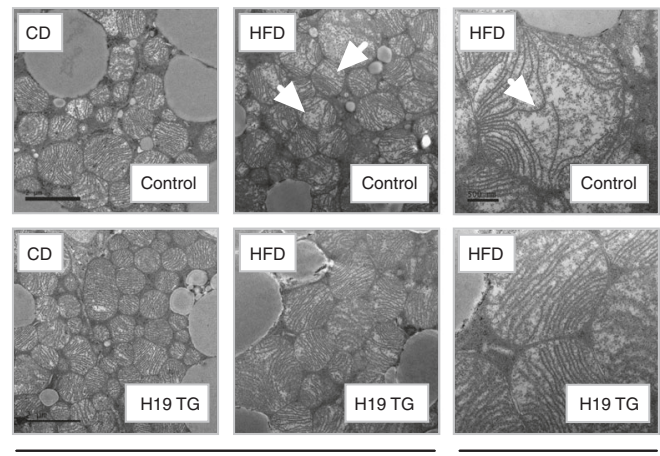

$10,000 x$

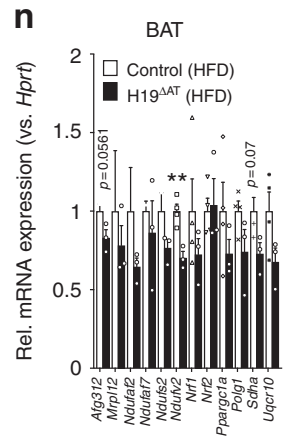

Mitochondrial biogenesis

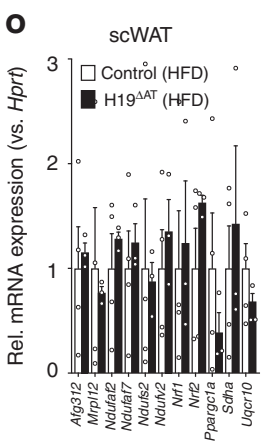

Mitochondrial biogenesis 
Female H19-DMR flDMR/flDMR were bred with male C57BL/6 expressing adiposeselective Adiponectin (Adipoq)-promoter driven Cre (H19-DMR wt/wt; Adipoq$\left.\mathrm{Cre}^{\mathrm{tg} / \mathrm{wt}}\right)$ to obtain H19-DMR ${ }^{\mathrm{flDMR} / \mathrm{wt}}$; Adipoq-Cretg/wt $\left(\mathrm{H} 19^{\Delta \mathrm{AT}}\right)$ and H19$\mathrm{DMR}^{\mathrm{flDMR} / \mathrm{wt}}$; and Adipoq-Cre ${ }^{\mathrm{wt} / \mathrm{wt}}$ control littermates (Control to $\mathrm{H} 19^{\Delta \mathrm{AT}}$ ). Only male, non-randomized mice were used with the experimentator not blinded to the genotype.

Mouse RNA isolation. RNA from indicated tissues and primary adipocytes was isolated using Trizol ${ }^{\circledast}$ according to manufacturer's protocols for total RNA isolation. For subcellular localization analyses, RNAs were purified from nuclear and cytoplasmic fractions obtained using the PARIS kit (Ambion) according to manufacturer's protocol.

Deep RNA-sequencing procedure. (1) For $4{ }^{\circ} \mathrm{C}$ versus $22^{\circ} \mathrm{C}$ and $\mathrm{CD}$ versus HFD BAT samples: library preparation and sequencing was performed at the Max Planck-Genome-centre Cologne, Germany. Following initial quality checks, $1 \mu \mathrm{g}$ total RNA of each sample was depleted for rRNA using NEBNext ${ }^{\oplus}$ rRNA depletion Kit (human/mouse/rat). Library preparation was performed with NEBNext Ultra ${ }^{\mathrm{m}}$ Directional RNA Library Prep Kit for Illumina (New England Biolabs). All libraries were sequenced in parallel on a HiSeq2500 instrument (Illumina) in $2 \times 100 \mathrm{bp}$ sequencing mode. (2) For siH19 and siCtrl transfected $1^{\circ}$ BAT and $1^{\circ}$ vWAT samples: library preparation and sequencing was carried out by the Core facility Genomics, Medical University of Vienna, Vienna, Austria. Briefly, sequencing libraries were prepared using the NEBNext ${ }^{\oplus}$ Ultra $^{\text {mat }}$ II RNA Library Prep Kit according to manufacturer's instructions and sequenced on Illumina NextSeq550 platforms. The resulting 75 bp single-end reads were quality-checked with FastQC (http://www.bioinformatics.babraham.ac.uk/projects/fastqc/), and low quality reads were removed using the fastq_quality_trimmer ("-t 20 -1 25" parameters) from the FASTX-Toolkit.

Deep RNA-sequencing data processing. RNA-Seq data were processed using the QuickNGS analysis pipeline ${ }^{60}$, version 1.2.7, based on Ensembl release 87. In brief, reads were mapped to the GRCm 38 assembly of the mouse genome using Tophat2, version 2.0.10, and reassembled with Cufflinks, version 2.1.1. Differential gene expression was analyzed using the DESeq2 package, version 1.10.1. The results were uploaded to the QuickNGS database and combined with multiple annotations using the biomaRt package. For $4{ }^{\circ} \mathrm{C}$ versus $22^{\circ} \mathrm{C}$ and $\mathrm{CD}$ versus HFD BAT samples, $n=2$ per condition and for siH19 and siCtrl transfected $1^{\circ}$ BAT and $1^{\circ}$ vWAT samples, $n=3$ per condition.

Gene set enrichment analysis. GSEA is a computational pathway analysis tool that determines if a given set of manually curated genes show statistically significant, concordant differences between two biological states (http://www. broadinstitute.org/gsea/index.jsp). A list of murine imprinted genes from www. geneimprint.com was used to construct gene sets representing all known PEGs and MEGs, respectively. Genes were first ranked based on real value using the weighted signal-to noise metric. $p$-values and false discovery rates (FDR) for the enrichment score of each gene set were calculated based on 1000 gene set permutations.

Cultivation of PIBA cell lines. PIBA cell lines were generated in-house (s. below) and maintained in growth medium Dulbecco's modified Eagle's medium (DMEM, PAA) containing $4.5 \mathrm{~g} / \mathrm{l}$ glucose supplemented with $10 \%$ FCS, $5 \mathrm{mM}$ L-glutamine,
$0.1 \mathrm{mM}$ non-essential amino acids, $1 \mathrm{mM}$ sodium pyruvate and $1 \times$ penicillin/ streptomycin $(\mathrm{P} / \mathrm{S})$. Cells were grown to confluency in differentiation medium (DMEM, $20 \mathrm{nM}$ insulin, $1 \mathrm{nM}$ T3). Brown adipocyte differentiation was induced using differentiation medium supplemented with $0.125 \mathrm{mM}$ indomethacin, $2 \mathrm{mg} / 1$ dexamethasone, and $0.5 \mathrm{mM}$ isobutylmethylxanthine for 1 day and incubated in differentiation medium for 5 more days.

Generation of stable PIBA cell lines. PIBA cells were prepared from C57BL/6 mice based on principles described in Klein et al. ${ }^{61}$ with slight modifications. Interscapular BAT from postnatal day three newborn-mice were resected and minced in $500 \mu \mathrm{l}$ sterile PBS. Tissue pieces were subjected to collagenase digestion by adding $500 \mu \mathrm{l}$ digestion buffer $(123 \mathrm{mM} \mathrm{NaCl}, 5 \mathrm{mM}$ $\mathrm{KCl}, 1.3 \mathrm{mM} \mathrm{CaCl} 2,5 \mathrm{mM}$ glucose, $100 \mathrm{mM}$ HEPES, $1 \% \mathrm{P} / \mathrm{S}, 4 \% \mathrm{BSA}$ ) containing $1.5 \mathrm{mg} / \mathrm{mL}$ Collagenase A (Roche), followed by repeated cycles of vortexing for $10 \mathrm{~s}$ and incubation at $37^{\circ} \mathrm{C}$ for $30 \mathrm{~s}$ every $5 \mathrm{~min}$ until a single cell suspension was achieved. Cell suspensions were filtered through a $100 \mu \mathrm{m}$ screen and cells collected by centrifugation at $422.2 \times g$ for $5 \mathrm{~min}$. The cells were then resuspended in Dulbecco's modified Eagle's medium (DMEM) supplemented with $4.5 \mathrm{~g} / \mathrm{l}$ glucose, $20 \%$ fetal calf serum (FCS), $20 \mathrm{mM}$ HEPES, sodium pyruvate, L-glutamine, non-essential amino acids and 1\% P/S and cultured at $37^{\circ} \mathrm{C}$ with $5 \% \mathrm{CO}$. Media was changed every day until cells had reached $80 \%$ confluency and were passaged once. For immortalization cells were transfected with a plasmid encoding the SV40 large T-antigen using Lipofectamine 2000 transfection-reagent (Invitrogen) according to the manufacturer's instructions. PIBA cells were tested mycoplasma negative before experiments.

Isolation stromal-vascular fraction derived $\mathbf{1}^{\circ}$ adipocytes. To isolate depot-specific SVF cells, male C57BL/6 mice were sacrificed by $\mathrm{CO} 2$ or cervical dislocation. Next, interscapular BAT, posterior inguinal subcutanous (scWAT) and perigonadal visceral (vWAT) tissue of 6-8 weeks old male of indicated genotypes was removed and tissues transferred into serum-free DMEM/Ham's F-12 medium. To obtain sufficient cell numbers, BAT tissues were pooled according to genotype if needed. In a sterile environment, tissue medium was aspirated before cutting the adipose tissue into pieces until a homogenized consistency was achieved. Homogenized tissue was collected in a $50 \mathrm{ml}$ tube filled with $10 \mathrm{ml}$ serum-free DMEM/Ham's F-12 medium. To wash out cellular debris, $5 \mathrm{ml}$ of the lower phase without visible tissue chungs were aspirated, $5 \mathrm{ml}$ serum-free DMEM/Ham's F-12 medium added and in a final wash $5 \mathrm{ml}$ of the infranatant removed. For each scWAT and vWAT tube $1 \mathrm{mg} / \mathrm{ml}$ Collagenase II, 1 $5 \% \mathrm{BSA}, 6.6 \mathrm{ml}$ serum-free medium and $100 \mu \mathrm{l}$ DNase $(15 \mathrm{kU} / \mathrm{ml})$ were prepared. For each BAT tube the same substances as for scWAT and vWAT plus $240 \mu \mathrm{l}$ Dispase I $(50 \mathrm{U} / \mathrm{ml})$ were added. To each adipose tissue preparation, $10 \mathrm{ml}$ of the mixed enzyme solution were added. Next the tubes were placed in a $37^{\circ} \mathrm{C}$ water bath and shaken at $120 \mathrm{rpm} / \mathrm{min}$ for $15 \mathrm{~min}$. The tubes were vigorously shaken by hand before warming again at $37^{\circ} \mathrm{C}$ for another $15 \mathrm{~min}$ at $120 \mathrm{rpm} / \mathrm{min}$ For purification, the cell suspension was filtered through $100 \mu \mathrm{m}$ filters into a $50 \mathrm{ml}$ tube filled with $20 \mathrm{ml}$ DMEM/Ham's F-12 medium. The suspension was centrifuged for $5 \mathrm{~min}, 21^{\circ} \mathrm{C}$ and $200 \times \mathrm{g}$, supernatants discarded and pellets resuspended in $1 \mathrm{ml}$ DMEM/Ham's F-12 growth medium (DMEM/Ham's F12 medium plus $10 \%$ Fetal Calf Serum, $1 \%$ P/S, $0.1 \%$ Biotin and $0.1 \%$ pantothenic acid) and filled up to $10 \mathrm{ml}$. After centrifugation for $5 \mathrm{~min}, 21^{\circ} \mathrm{C}$ and $200 \times g$, the medium was aspirated up to $1 \mathrm{ml}$, the pellet resuspended and transferred into a $15 \mathrm{ml}$ tube. The tube was filled up to $13 \mathrm{ml}$ with growth medium and centrifuged for $5 \mathrm{~min}, 21^{\circ} \mathrm{C}$ and $200 \times g$. Next, supernatants were

Fig. 4 Fat-specific deletion of $\mathrm{H} 19$ sensitizes towards DIO-associated weight gains and impairments in energy expenditure. a Mating strategy for parental allele-of-origin specific deletion of the H19 DMR in adipose tissue. b Body weight of HFD-fed Control $(n=5)$ versus H19 $\triangle$ AT $(n=4)$ mice. A 2WA-B was applied to assess significance. c Glucose tolerance test of male, HFD-fed Control $(n=5)$ versus H19 $\Delta$ AT $(n=4)$ mice. $\mathbf{d}$ Insulin tolerance test of male, HFD-fed Control $(n=5)$ versus H19 $\triangle \mathrm{AT}(n=4)$ mice. e Energy expenditure in male, HFD-fed Control $(n=5)$ versus H19 $\Delta$ AT $(n=4)$ mice. $\mathbf{f}$ Oxygen consumption in male, HFD-fed Control $(n=5)$ versus H19 $\triangle$ AT $(n=4)$ mice. d-f A 2WA-RM test with repeated measures was applied to assess statistical significance. $\mathbf{g}$ RER in male, HFD-fed Control $(n=5)$ versus H19 $\triangle$ AT $(n=4)$ mice. $\mathbf{h}$ Tissue/body weight ratio in male, HFD-fed Control $(n=5)$ versus H19 ${ }^{\Delta A T}(n=4)$ mice. An unpaired, two-tailed Student's $t$-test was applied to assess significance. $\mathbf{i}, \mathbf{j}$ Automated quantification of mitochondrial area (i) and perimeters $(\mathbf{j})$ in BAT across diets and genotypes (total mitochondria numbers in brackets). An unpaired, two-tailed Student's t-test was applied to assess significance. $\mathbf{k}$ Representative electron microscopy images from BAT mitochondria across diets and genotypes with magnifications indicated under panel. White arrows depict cristae architectures. Scale bar, $2 \mu \mathrm{m}(6000 \times)$ or $500 \mathrm{~nm}(10,000 \times)$ I BAT expression of indicated mRNAs in HFD-fed H19 TG $(n=3)$, HFD-fed Control $(n=4)$, and CD-fed Control $(n=5)$ mice. $\mathbf{m}$ scWAT expression of indicated mRNAs in HFD-fed H19 TG ( $n=3)$, HFD-fed Control $(n=4)$, and CD-fed Control $(n=5)$ mice. I, $\mathbf{m}$ A One-Way ANOVA plus Bonferroni post test was applied to assess statistical significance. (a) $=$ Significance versus CD-fed Control, $(b)=$ Significance versus HFD-fed Control. $\mathbf{n}$ BAT expression of indicated mRNAs in HFD-fed Control $(n=4)$ versus HFD-fed H19 $\triangle \mathrm{AT}(n=3)$ mice. o scWAT expression of indicated mRNAs in HFD-fed Control $(n=4)$ versus HFD-fed H19 $\triangle \mathrm{AT}(n=3)$ mice. An unpaired, two-tailed Student's $t$-test was applied to assess significance between genotypes. ${ }^{\star} p<0.05,{ }^{\star \star} p<0.01,{ }^{\star \star \star} p<0.001$. If applicable $p$-values are indicated within the panel 
aspirated and pellets resuspended in $1 \mathrm{ml}$ Erythrocyte Lysis Buffer for $5 \mathrm{~min}$ The tube was filled up to $13 \mathrm{ml}$ and centrifuged for $5 \mathrm{~min}, 21^{\circ} \mathrm{C}$ and $200 \times g$. The supernatant was aspirated up to $1 \mathrm{ml}$ solution. In the remaining $1 \mathrm{ml}$, the pellet was resuspended and the solution filtered using a $30-\mu \mathrm{m}$ filter wetted with $500 \mu \mathrm{l}$ growth medium. After filtration, the filter was rinsed with $500 \mu \mathrm{l}$ growth medium and isolated cells counted using a Neubauer Counting Chamber to seed 50,000 cells/well in flat bottom 24-well-plates.
Induction of SVF adipogenesis. To induce commitment of SVFs into mature adipocytes, freshly prepared $0.05 \%$ Insulin, $0.005 \%$ Dexmethasone, $0.001 \%$ Rosiglitazone, and $0.05 \%$ 3-Isobutyl-1-methylxanthin (IBMX, scWCAT and vWAT) or $0.1 \%$ Indomethazine, $0.001 \%$ Triiodothyronine (BAT) in growth medium

(induction medium) were added to confluent cells. After $48 \mathrm{~h}$ of induction, differentiation was initiated using freshly prepared $0.001 \%$ Rosiglitazone (scWAT and vWAT) or $0.001 \%$ Triiodothyronine (BAT) in growth medium (differentiation
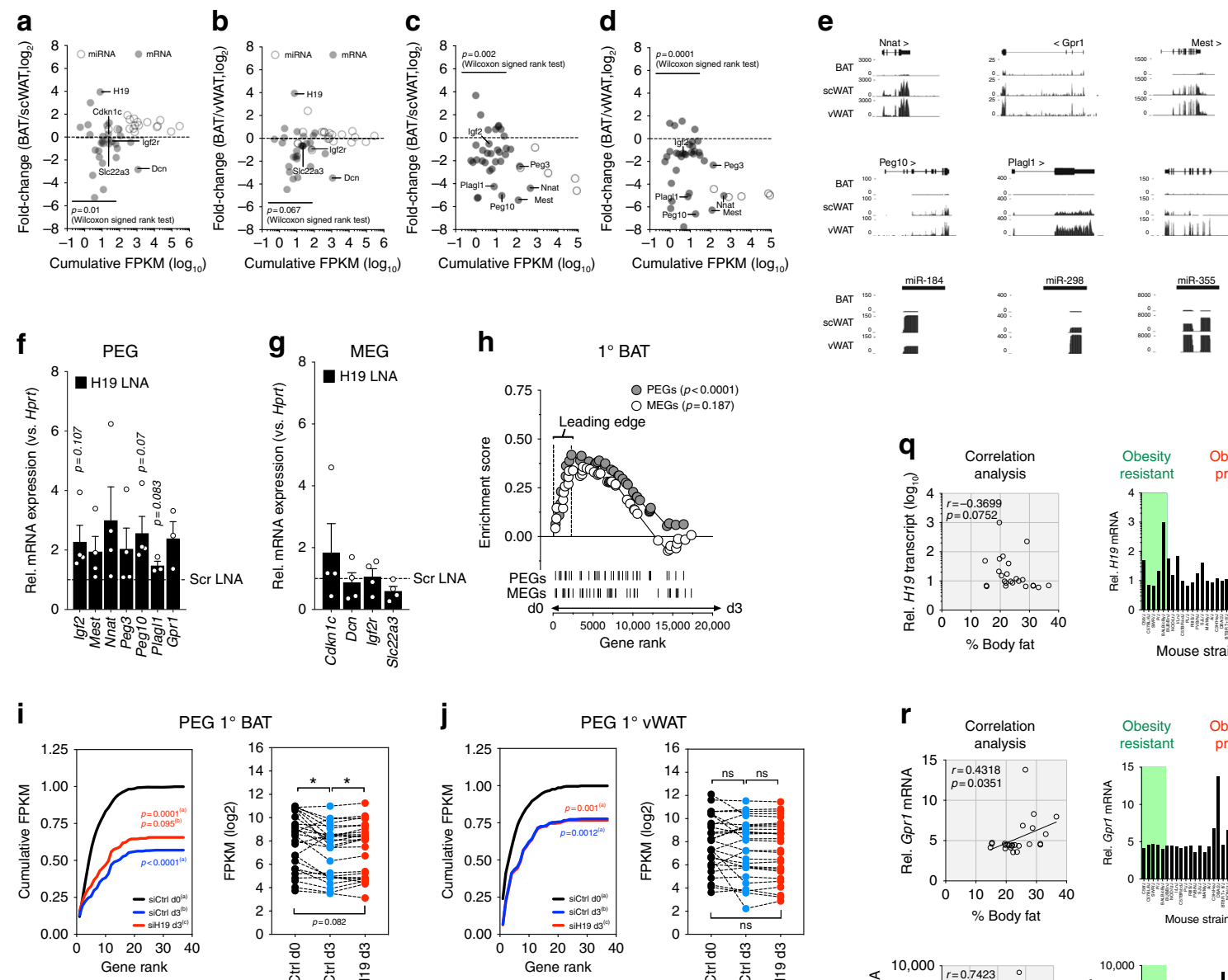

PEG $1{ }^{\circ}$ BAT
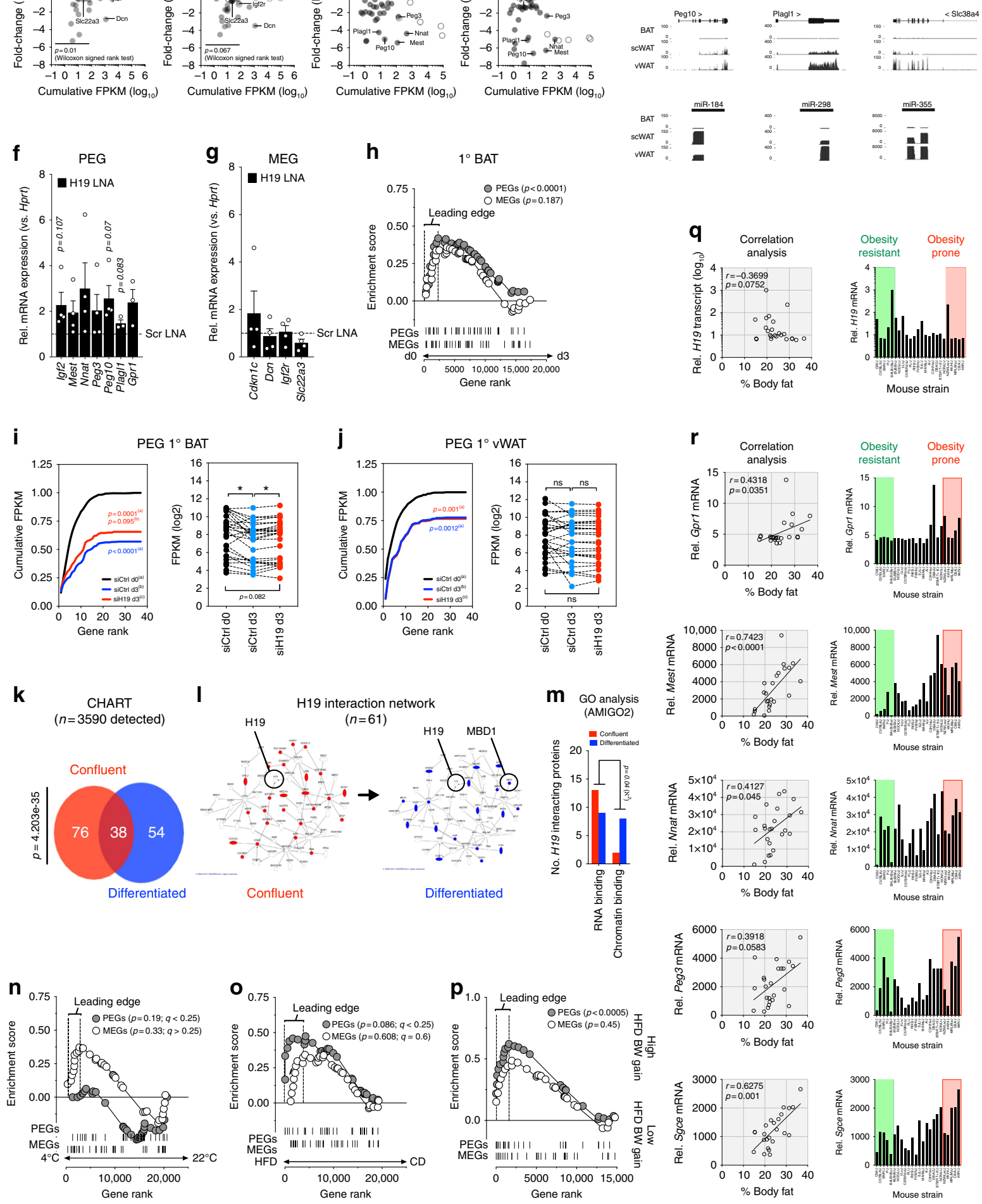
medium). Differentiation was achieved after 3-4 days of incubation in differentiation medium.

\begin{abstract}
LNA-mediated gene knockdown of primary adipocytes. Primary brown adipocytes were derived from depots-specific SVFs as described above. Cells were grown until confluency in growth medium. For H19 inhibition custom-made LNA GapmeRs were transfected after cells reached confluency (GapmeR sequences are provided in Supplementary Data 6). Lipofectamine 2000 was diluted 1:62.5 in OptiMEM. For a final concentration of $25 \mathrm{nM}$, respective LNAs (stock $10 \mu \mathrm{M}$ ) were diluted 1:50 in Opti-MEM. Both solutions were incubated for $5 \mathrm{~min}$ at RT. LNA and Lipofectamine solutions were mixed at equal volumes and incubated for $20 \mathrm{~min}$ at RT. The cells were washed with pre-warmed PBS and $750 \mu \mathrm{l}$ of growth medium without $\mathrm{P} / \mathrm{S}$ added to each well. A volume of $250 \mu \mathrm{l}$ of LNA/Lipofectamine mix was added and cells incubated for $24 \mathrm{~h}$ at $37^{\circ} \mathrm{C}$ and $5 \% \mathrm{CO}_{2}$ before changing medium to induction medium.
\end{abstract}

siRNA-mediated gene knockdown of primary adipocytes. SiRNAs targeting H19 (siH19, oligo n253571) or non-targeting Control (siCtrl, Cat. No. 4390843; both $100 \mathrm{nmol} / \mathrm{l}$, Invitrogen) were delivered into $1^{\circ} \mathrm{BAT}$ or vWAT adipocytes by Amaxa nucleofection (Lonza Bioscience) according to manufacturer's recommendations. The cells were utilized $48-72 \mathrm{~h}$ after transfection. SiRNA sequences are provided in Supplementary Data 6.

Immunoblot analysis. For protein isolation, the medium of cells was aspirated and the plates were stored on ice. The cell monolayer was washed gently one time with ice-cold PBS. Excess PBS was aspirated. A volume of $50 \mu \mathrm{l}$ of RIPA lysis buffer with inhibitors was added to each well of the 24-well plate. RIPA buffer was composed of $50 \mathrm{mM}$ Tris-HCL pH 7.5, $150 \mathrm{mM}$ NaCL $1 \mathrm{mM}$ EDTA, $0.1 \%$ sodium deoxycholate, $1 \%$ NP- $40,1 \times$ protease inhibitor and $1 \times$ phosphatase inhibitor. An icecold cell scraper was used to scrape the cells. The lysate was transferred to $1.5 \mathrm{ml}$ Eppendorf tubes. The samples were snap-frozen in liquid nitrogen and thawed on ice for three repeated cycles. After $10 \mathrm{~min}$ of centrifugation at $12,000 \times \mathrm{g}$ and $4{ }^{\circ} \mathrm{C}$ the supernatant was transferred to fresh tubes and stored on $-80^{\circ} \mathrm{C}$. Protein concentration was determined using the bicinchonoinic acid method. Samples were separated by SDS-PAGE after being mixed with $4 \times$ Laemmli Sample Buffer containing $10 \% \beta$-mercaptoethanol and heated to $95^{\circ} \mathrm{C}$ for $5 \mathrm{~min}$. Afterwards, proteins were transferred to nitrocellulose membrane for incubation with primary antibodies raised against PGC-1A (sc-13067, Santa Cruz 1:500), UCP1 (sc-6528, Santa Cruz, 1:500). Calnexin (208-880, Calbiochem, 1:5000) served as loading control. Uncropped scans of the blots can be found as a Supplementary Figure in the Supplementary Information.

Oil Red O Staining. ORO solution was prepared by dissolving $0.3 \mathrm{~g}$ ORO dye in $60 \mathrm{ml}$ isopropanol in the dark overnight at room temperature. Afterwards, $40 \mathrm{ml}$ $\mathrm{dH} 2 \mathrm{O}$ was added and the solution filtered. In a fume hood the media of the cell plates was aspirated and plates rinsed with $2 \mathrm{ml}$ of sterile PBS per well. Next, PBS was aspirated and $1 \mathrm{ml}$ of $10 \%$ formalin added. Cells were incubated for $1.5 \mathrm{~h}$ at room temperature, excess formalin removed, wells washed with $2 \mathrm{ml}$ PBS and stained with $1 \mathrm{ml}$ ORO staining solution for $2 \mathrm{~h}$. Before image acquisition, the wells were washed twice with $\mathrm{dH} 2 \mathrm{O}$ for $5 \mathrm{~min}$.

Oxygen consumption rates and glycolytic activity. SVF from indicated adipose tissue depots were seeded into Agilent Seahorse XFe96 Bioanalyzer microplates. Per well 50,000 cells were seeded and incubated in DMEM/Ham's F-12 medium plus $10 \%$ Fetal Calf Serum, $1 \%$ P/S, 0.1\% Biotin and 0.1\% Pantothenic acid (Growth medium) at $37^{\circ} \mathrm{C}$ and $5 \% \mathrm{CO} 2$ at a standard incubator until confluency is reached For $\mathrm{H} 19$ inhibition cells were transfected as described above directly in the Seahorse setup. For this, the cells were washed with pre-warmed PBS and $75 \mu \mathrm{l}$ of growth medium w/o P/S added to each well. A volume of $25 \mu \mathrm{l}$ of LNA/Lipofectamine mix was added and cells incubated for $24 \mathrm{~h}$ at $37^{\circ} \mathrm{C}$ and $5 \% \mathrm{CO}_{2}$ before changing medium to induction medium. To induce commitment of SVFs into mature adipocytes directly within the Seahorse microplates, freshly prepared $0.05 \%$ Insulin, $0.005 \%$ Dexmethasone, $0.001 \%$ Rosiglitazone, and $0.05 \%$ IBMX (scWCAT and vWAT) or $0.1 \%$ Indomethazine, $0.001 \%$ Triiodothyronine (BAT) in growth medium (induction medium) were added. After $48 \mathrm{~h}$ of induction, differentiation was initiated using freshly prepared $0.001 \%$ Rosiglitazone (scWAT and vVAT) or $0.001 \%$ Triiodothyronine (BAT) in growth medium (differentiation medium). Differentiation was achieved after 3-4 days of incubation in differentiation medium. For each seahorse plate the corresponding calibration plate was prepared $24 \mathrm{~h}$ prior to experiments using $200 \mu \mathrm{l} \mathrm{XF} \mathrm{Seahorse} \mathrm{Calibrant} \mathrm{Agilent} \mathrm{per} \mathrm{well.} \mathrm{The} \mathrm{plate}$ was incubated for $24 \mathrm{~h}$ in a non-CO2 incubator at $37^{\circ} \mathrm{C}$ and the instrument set to $37^{\circ} \mathrm{C} 24 \mathrm{~h}$ prior to the experiment. One hour before measuring the plate, it was washed with PBS and the medium changed according to the corresponding experiment analyte kits (MitoStressKit or GlycoStressKit, provided by the manufacturer). Prior to measurement, calibration was started using calibration plates, measuring $\mathrm{O} 2$ and $\mathrm{pH}$ LED Value/emission/Initial reference Delta for each well. After calibration cartridges were kept within the machine and measurement of adipocyte-containing microplates commenced. Measurement parameters were: Mix: $3 \mathrm{~min}$, wait $0 \mathrm{~min}$, measure $3 \mathrm{~min}$ with each reagent's effect assessed within 3 (MitoStressKit) or 4 measurement cycles (GlycoStressKit) with a total duration of 18 or 24 min per reagent injection. All measurements started with measuring basal values, followed by injection of Oligomycin, FCCP and Rotenone + Antimycin A (MitoStressKit) or Glucose, Oligomycin and 2-Deoxy-Glucose, GlycoStressKit). Coupling efficiencies were calculated as reported recently ${ }^{62}$

For MitoStress Kits, corresponding media were prepared shortly before the experiment and consisted of Basal Seahorse Medium supplemented with $25 \mathrm{mM}$ Glucose, $1 \mathrm{mM}$ Glutamine, $2 \mathrm{mM}$ Sodium Pyruvate, set to $\mathrm{pH}=7.4$ and filtered sterile. Per Plate ca. $25 \mathrm{ml}$ of MitoStress Medium were needed and Seahorse cell plates were changed to $180 \mu \mathrm{l}$ MitoStress medium $1 \mathrm{~h}$ prior to calibration in a non$\mathrm{CO} 2$ incubator at $37^{\circ} \mathrm{C}$. The calibration plate possessed a cartridge having 4 pockets per well. Before the measurement pocket A was filled with $20 \mu \mathrm{l} 10 \mu \mathrm{M}$ Oligomycin, pocket B with $22 \mu \mathrm{l} 10 \mu \mathrm{M}$ FCCP and pocket C with $25 \mu \mathrm{l} 5 \mu \mathrm{M}$ Antimycin A and Rotenone. For the GlycoStressKit, the cell plate was washed with PBS and media changed to filtered $180 \mu \mathrm{l}$ GlycoStress medium. GlycoStress medium consisted of Basal Seahorse Medium supplemented with $1 \mathrm{mM}$ Glutamine and $2 \mathrm{mM}$ Sodium Pyruvate, set to $\mathrm{pH}=7.4$ and stored for $1 \mathrm{~h}$ in a non-CO2 incubator at $37^{\circ} \mathrm{C}$. The calibration plate possessed a cartridge having 4 pockets per well. Shortly before the measurement pocket A was filled with $20 \mu \mathrm{l} 10 \mathrm{mM}$ Glucose, pocket B with $22 \mu \mathrm{l} 10 \mu \mathrm{M}$ Oligomycin and pocket C with $25 \mu \mathrm{l} 50 \mathrm{mM}$ 2-Deoxy-Glucose.

Glucose tolerance test \& insulin tolerance test. At the time of performing insulin tolerance test (ITTs), mice were 12 weeks of age and 9 weeks exposed to CD or HFD feeding. The ITT was carried out in random-fed mice at 9-10 am in the morning in fresh cages with bedding, free access to drinking water but no food. After determining basal blood glucose levels $(0 \mathrm{~min})$, each animal received $0.75 \mathrm{U} /$ $\mathrm{kg}$ of body weight of insulin (Actrapid; Novo Nordisk). Blood glucose levels were recorded after 15, 30 and $60 \mathrm{~min}$ in male $\mathrm{H}_{1} 9^{\Delta \mathrm{AT}}(\mathrm{CD}, n=5)$, Control (Control for H19 ${ }^{\Delta \mathrm{AT}}, \mathrm{CD}, n=5$ ), H19 ${ }^{\triangle \mathrm{AT}}$ (HFD, $\left.n=4\right)$, Control (Control for H19 ${ }^{\Delta \mathrm{AT}}$, HFD, $n=5$ ), H19 TG (CD, $n=6$ ), Control (Control for H19 TG, CD, $n=5)$, H19 TG (HFD, $n=6$ ), and Control (Control for H19 TG, HFD, $n=4$ ) mice. At the time of performing glucose tolerance test (GTTs), mice were 13 weeks of age and

Fig. 5 Repression of BAT paternal monoallelic gene expression by the lincRNA H19. a Plot of expression fold-changes of maternally expressed genes (MEGs) in BAT versus a scWAT $\mathbf{b} v W A T$. $\mathbf{c}$ Plot of expression fold-changes of paternally expressed genes (PEGs) between BAT versus $\mathbf{c}$ scWAT $\mathbf{d}$ vWAT. A Wilcoxon matched-pairs signed rank test was used to assess statistical significance for up- or downregulation of PEGs/MEGs (e) UCSC Genome Browser showing PEG abundances in BAT, scWAT, and vWAT. f, $\mathbf{g}$ Expression of indicated PEGs (f) or MEGS (g) in BAT $1^{\circ}$ adipocytes transfected with scr or H19 LNA. f, $\mathbf{g}$ A paired, two-tailed Student's $t$-test was used to assess significance across $n=4$ experiments, $n=3$ technical replicates each. $\mathbf{h}$ GSEA showing downregulation of PEGs but not MEGs (list from www.geneimprint.com) during $1^{\circ}$ BAT differentiation. $\mathbf{i}, \mathbf{j}$ Cumulative distribution frequency (left) and abundances (right) of PEGs in $\mathbf{i} 1^{\circ} \mathrm{BAT}$ and $\mathbf{j} 1^{\circ} \mathrm{vWAT}$ in siCtrl transfected adipocyte progenitors at do (black) versus differentiated siCtrl (blue) and siH19-transfected (red) $1^{\circ}$ adipocytes at d3. k Quantification and overlap of H19 co-immunoprecipitating proteins in confluent (red) and differentiated (blue) PIBA cells determined by CHART-MS. Pulldown of H19-interacting proteins was performed using six H19-specific antisense/sense oligonucleotides in $n=3$ replicates. I Illustration of H19 interaction network comprising $n=61$ proteins generated using Ingenuity Pathway Analysis. Red and blue nodes depict proteins co-immunoprecipitating in confluent and differentiated cells, respectively. $\mathbf{m}$ AMIGO2 GO classification of H19 interactors across cellular states. n-p GSEA analysis of PEG gene ranks in mice $\mathbf{n}$ exposed to $4^{\circ} \mathrm{C}$ cold stress for $24 \mathrm{~h}$, o HFD feeding, or $\mathbf{p}$ exhibiting different susceptibility to DIOevoked weight gains ${ }^{53}$. q, r Correlation of WAT (q) H19 or (r) PEG abundances versus a ranked list of 24 obesity-prone and -resistant non-isogenic mouse strains. H19/PEG abundances were from (MOE430 V2)-Adipose (www.biogps.org54) and body composition after HFD feeding defined as obesityresistant versus obesity-prone strains ${ }^{65}$. Significance of association between expression versus (\%) body fat was determined using Spearman's correlation analysis. ${ }^{\star} p<0.05,{ }^{\star \star} p<0.01,{ }^{\star \star \star} p<0.001$. If applicable additional $p$-values are indicated within the panel 
10 weeks exposed to CD or HFD feeding. GTT was carried out at 12 am after a $6 \mathrm{~h}$ fast starting in the morning. After determining basal blood glucose levels $(0 \mathrm{~min})$, animals received an intraperitoneal bolus of $2 \mathrm{~g}$ glucose per kilogram of body weight (20\% glucose, Delta select). Blood glucose levels were determined $15,30,60$, and 120 min after injection using an automatic glucose monitor (Contour, Bayer Diabetes Care) in male H19 $9^{\Delta \mathrm{AT}}(\mathrm{CD}, n=5)$, Control (Control for H19 $9^{\Delta \mathrm{AT}}$, CD, $n=5$ ), H19 $9^{\Delta \mathrm{AT}}$ (HFD, $n=4$ ), Control (Control for H19 ${ }^{\Delta \mathrm{AT}}$, HFD, $n=5$ ), H19 TG (CD, $n=6$ ), Control (Control for H19 TG, CD, $n=5$ ), H19 TG (HFD, $n=5$ ), Control (Control for H19 TG, HFD, $n=5$ ) mice. Animals were excluded from analysis that showed no increase/decrease of blood glucose levels after i.p. injection of glucose (GTT) or insulin (ITT), respectively, assuming injection outside of the peritoneal cavity as required for the assay.

Indirect calorimetry (PhenoMaster). Upon indirect calorimetry measurements, mice of all genotypes and diets were 16 weeks of age and 13 weeks exposed to $C D$ or HFD. Metabolic measurements were obtained using a PhenoMaster System (TSE Systems). For this, five days before analysis, the mice were placed alone in training cages, identical to the 7.1-l chambers of the PhenoMaster open circuit calorimetry system and continued to receive respective diets (CD, HFD) throughout training and data acquisition. Diets and water were provided ad libitum in the appropriate devices and food intake measured by the built-in automated instruments. Parameters of indirect calorimetry were measured initially for $96 \mathrm{~h}$ at $22{ }^{\circ} \mathrm{C}$ (warm measurement). Subsequently, temperatures within the PhenoMaster setup were reduced to $4{ }^{\circ} \mathrm{C}$ (cold measurement) and data acquired for $96 \mathrm{~h}$. At the end, NE was administered at $4{ }^{\circ} \mathrm{C}$ intraperitoneally at a final concentration of $1 \mathrm{mg} /$ $\mathrm{kg}$ and measurements continued for another $24 \mathrm{~h}$ (NE measurement) to measure maximally activated BAT uncoupling effects. Mean values for each time of day were calculated and plotted for warm, cold and NE measurement in male H19 $\triangle \mathrm{AT}$ $\left(\mathrm{CD}, n=5\right.$ ), Control (Control for H19 $9^{\Delta \mathrm{AT}}, \mathrm{CD}, n=5$ ), Male H19 ${ }^{\Delta \mathrm{AT}}$ (HFD, $n=4$ ), Control (Control for H19 ${ }^{\triangle \mathrm{AT}}$, HFD, $\left.n=5\right)$, H19 TG (CD, $n=5$ ), Control (Control for H19 TG, CD, $n=4$ ), H19 TG (HFD, $n=4$ ), Control (Control for H19 TG, HFD, $n=5$ ) mice.

mRNA isolation and quantitative RT-PCR (qPCR) analysis. Total RNA was isolated from primary adipocytes and tissues using peqGOLD TriFast (PEQLAB Biotechnologie). mRNA was reverse transcribed into complementary DNA using EuroScript reverse transcriptase (Eurogentec). Abundances of mRNAs/lncRNAs were quantified by TaqMan Assay on Demand Kits (Applied Biosystems) according to the manufacturer's protocol if not indicated otherwise. Abundances of Adipoq, Afg312, CD24, CD29, Cdkn1c, Cebpa, Cebpb, Cox4i1, Cox7a1, Cox8b, Drp1, Elovl3, Fabp4, Fis1, Gata2, H19, hnNctc1, Igf2r, Ly6a, Mff, Mfn1, Mfn2, Mrpl12, Nctc1, Ndufaf2, Ndufaf7, Ndufs2, Ndufv2, Nrf1, Nrf2, Opa1, Peg10, Plagl1, Polg, Ppara, Pparg, Pgcla, Prdm16, Sdha, Slc22a3, Tgfb2, Ucp1, and Uqcr10 were quantified using SYBR methodology using Select Master Mixes (Thermo Fisher). The relative abundance of mRNAs was calculated using comparative methods $\left(2^{-\delta \delta C T}\right)$ according to ABI Relative Quantification Methods. Transcript levels of mRNAs were normalized to hypoxanthine phosphoribosyltransferase 1 (Hprt1) expression; Hprt abundances were unaffected across all experimental conditions. SYBR primer sequences are provided in Supplementary Data 6.

Immunohistochemistry. Resected BAT, scWAT, vWAT, and liver specimens were incubated at $4{ }^{\circ} \mathrm{C}$ overnight in $4 \%$ paraformaldehyde, embedded in paraffin and sliced according to standard protocols. Haematoxylin and eosin (HE) stainings were carried out after deparaffination as described ${ }^{63}$

\begin{abstract}
Automated adipocyte quantification. HE stainings were prepared as described above. An automated workflow was devised to segment and measure adipocytes from the tissue images. First, the interactive learning and segmentation toolkit ilastik (www.ilastik.com) was used to train tissue-specific pixel-level classifiers on the RGB photomicrographs. For classification of BAT three classes were defined (membrane, vacuole, nucleus) and a classifier using a set of eleven optimized features selected by the filter method was trained based on a manually labeled training data set. For images from scWAT and vWAT a unified classifier was trained with just two classes (membrane and vacuole) and using all available features. Application of the classifiers to the respective image sets resulted in two or three class probability maps, which were fed in Cellprofiler to segment and individually measure single adipocytes. The vacuole probability map was smoothed and automatically thresholded. The obtained binary vacuole mask was subjected to morphological closing to fill small holes. The result was used to mask the original vacuole probability image, which then was used to perform intensity/probability based cell segmentation by means of the Cellprofiler Identify Primary Objects module. The cell masks were expanded until touching their neighboring cells and cells intersecting with the image border before expansion were excluded. Size and shape parameters of the remaining cells were measured.
\end{abstract}

Electron microscopy and mitochondrial morphometry. For the fixation of the tissues the specimen were cut into small $\left(\sim 1 \mathrm{~mm}^{3}\right)$ pieces and stored in fresh fixative (10 ml Caco-buffer, $1.6 \mathrm{ml}$ of $25 \%$ Glutardialdehyd, $5 \mathrm{ml}$ of $8 \%$ PFA in $\mathrm{H} 2 \mathrm{O}$, filled up to $20 \mathrm{ml}$ with DEPC-treated $\mathrm{H} 2 \mathrm{O}$ with $\mathrm{pH}$-value adjusted to 7.3 ) for
$16-24 \mathrm{~h}$ at $4{ }^{\circ} \mathrm{C}$. For embedding of the fat tissue samples were washed $4 \times$ for $20 \mathrm{~min}$ in $0.1 \mathrm{M}$ Caco-buffer $(\mathrm{pH}=7.2-7.3)$ at $4^{\circ} \mathrm{C}$. The samples were stored for $2 \mathrm{~h}$ in $2 \%$ $\mathrm{OsO}_{4}$ in $0.1 \mathrm{M} \mathrm{Caco-buffer}(\mathrm{pH}=7.2-7.3)$ at $4^{\circ} \mathrm{C}$ in the dark. Afterwards, samples were washed in $0.1 \mathrm{M}$ Caco-buffer $(\mathrm{pH}=7.2-7.3) 4 \times$ for $20 \mathrm{~min}$ at $4^{\circ} \mathrm{C}$. For dehydration samples were stored in ice-cold $50 \% \mathrm{EtOH}$ for $20 \mathrm{~min}$ at $4^{\circ} \mathrm{C}$. Next, samples were kept in ice-cold $70 \% \mathrm{EtOH}$ at $4{ }^{\circ} \mathrm{C}$ overnight. The next day, samples were stored for $20 \mathrm{~min}$ in ice-cold $90 \% \mathrm{EtOH}$ at $4{ }^{\circ} \mathrm{C}$. After that, the samples were stored $3 \times$ for $20 \mathrm{~min}$ in ice-cold $100 \% \mathrm{EtOH}$ at $4{ }^{\circ} \mathrm{C}, 20 \mathrm{~min}$ in equal volumes of Propylenoxid:EtOH at $4{ }^{\circ} \mathrm{C}$ and $2 \times 20 \mathrm{~min}$ in pure Propylenoxid at $4{ }^{\circ} \mathrm{C}$. For transfering the samples to epon (20 g Epoxy, $11 \mathrm{~g}$ DDSA, $9 \mathrm{~g} \mathrm{NMA}, 0.8 \mathrm{~g}$ DMP30) samples were stored for $5 \mathrm{~h}$ in equal volumes of Epon:Propylenoxid at $4^{\circ} \mathrm{C}$. Moreover, samples were incubated in 3:1 volumes of Epon:Propylenoxid overnight at $4{ }^{\circ} \mathrm{C}$. Fresh epon was used first to store the samples at $4{ }^{\circ} \mathrm{C}$ during the day as well as overnight at $4^{\circ} \mathrm{C}$. On the next day, samples were immersed in fresh epon for $2 \mathrm{~h}$ with opened lid. The final embedding was performed in a flat form in fresh epon for $72 \mathrm{~h}$ at $62{ }^{\circ} \mathrm{C}$. After embedding the samples, semi-thin sections $(500 \mathrm{~nm})$ were cut with a Leica UC6 ultramicrotome to check the quality of the sample. Afterwards, ultrathin sections were produced. The sections were fit to the grid and contrasted. The grid was incubated for $15 \mathrm{~min}$ in $1.5 \%$ aqueous Uranylacetate, washed $5 \times$ in distilled $\mathrm{H}_{2} \mathrm{O}$, incubated again for $4 \mathrm{~min}$ in lead citrate and washed $5 \times$ in distilled $\mathrm{H}_{2} \mathrm{O}$. Finally, samples were checked with electron microscope Jeol 2100 Plus equipped with a Gatan camera.

Study participants. Paired samples of scWAT and omental vWAT were obtained from 169 individuals ( 113 women, 56 men). The age ranged from 19 to 88 years and BMIs from 18.9 to $73.1 \mathrm{~kg} / \mathrm{m}^{2}$. All adipose tissue samples were collected during laparoscopic abdominal surgery ${ }^{64}$. Adipose tissue was immediately frozen in liquid nitrogen and stored at $-80^{\circ} \mathrm{C}$. The study was approved by the Ethics Committee of the University of Leipzig (approval no: 159-12-21052012), and performed in accordance to the declaration of Helsinki. All subjects gave written informed consent before taking part in this study. BMI was calculated by weight $(\mathrm{kg})$ divided by square of height $(\mathrm{m})$. All baseline blood samples were collected between 8 and 10 am after an overnight fast. Plasma glucose, HbAlc, HDL-, LDL-cholesterol, free fatty acids, and triglycerides were measured in an automated analyzer (Cobas 8000, Roche Diagnostics, Mannheim, Germany).

Analysis of H19 expression. RNA from human scWAT and vWAT was extracted by using RNeasy Lipid Tissue Mini Kit (Qiagen, Germany). Quantity and integrity of RNA was monitored via spectrophotometry using NanoVue plus (GE Healthcare, Germany). A volume of $1 \mu \mathrm{g}$ total RNA from scWAT and vWAT adipose tissue were reverse transcribed with standard reagents (Life technologies, Germany). cDNA was processed for TaqMan probe-based quantitative real-time polymerase chain reaction (qPCR) using the QuantStudio 6 Flex Real-Time PCR System (Life technologies, Germany). Expression of $H 19$ was calculated by standard curve method and normalized to the expression of hypoxanthine guanine phosphoribosyltransferase 1 (HPRT1) as housekeeping gene. TaqMan probes (Life technologies, Germany) for H19 (Hs00262142_g1) and HPRT1 (Hs01003267_ml) span exon-exon boundaries to improve the specificity of the qPCR.

CHART-MS. For ChART-MS, PIBA cells were seeded and either only grown to confluency or induced to differentiate. The cells were washed with $1 \times$ PBS and fixed with $1 \%$ Formaldehyde for $10 \mathrm{~min}$ and fixation quenched with $0.125 \mathrm{M}$ Glycine. The cells were washed with ice-cold $1 \times$ PBS, scraped off and centrifuged for $5 \mathrm{~min}$ at $100 \times g$. The supernatant was aspirated and cells stored on $-80^{\circ} \mathrm{C}$. The cells were lysed in $400 \mu \mathrm{l}$ lysis buffer, resuspended and stored on ice for $5 \mathrm{~min}$. This step was repeated and $0.1 \%$ of NP-40 was added to the lysis buffer. $1 \mathrm{ml}$ of Sucrose buffer was pipetted into a new tube. The lysed cells (max. $400 \mu \mathrm{l})$ were carefully pipetted to the center of the sucrose buffer and samples centrifuged for $10 \mathrm{~min}$ at $7000 \times g$ at $4{ }^{\circ} \mathrm{C}$. The lysis step with only $400 \mu \mathrm{l}$ of lysis buffer and the sucrose step was repeated. After centrifugation, supernatants were discarded and nuclei washed with $1 \mathrm{ml} \mathrm{PBS}$. The tube was centrifuged for $1 \mathrm{~min}$ at $7000 \times g$, supernatants discarded and $1 \mathrm{ml}$ of PBS added. Suspensions were resuspended and vortexed until achieving a single nucleus suspension. For crosslinking of nuclei $2 \%$ formaldehyde was added for $30 \mathrm{~min}$, tubes vortexed and stored for $30 \mathrm{~min}$ on a wheel at room temperature. For quenching $0.125 \mathrm{M}$ Glycine was added and samples centrifuged for $1 \mathrm{~min}$ at $7000 \times g$ at $4{ }^{\circ} \mathrm{C}$. Supernatants were discarded and nuclei washed with $1 \mathrm{ml}$ PBS. The samples were centrifuged at $7000 \times g$ for $1 \mathrm{~min}$. Washing was repeated with $2 \mathrm{~min}$ of centrifugation in between. Supernatants were discarded and $300 \mu \mathrm{l}$ nuclear lysis buffer and RNAse inhibitor added. Samples were vortexed and incubated for $5 \mathrm{~min}$ on ice. PBS and protease inhibitor were added in equal volumes to nuclear suspension and the suspensions vortexed. To lyse residual nuclei a tip sonicator was used with the following conditions: $3 \times$ cycles $10 \%$, $3 \times 10$ s, POWER $10 \%$. The suspension was transferred into Diagenode TPX microtubes and tubes sonicated with following conditions: Amplitude: $35 \%, 15 \mathrm{~s}$ ON, $15 \mathrm{~s}$ OFF, 36 cycles, time: $9 \mathrm{~min}$ for appropriate shearing of mature brown adipocyte chromatin. Afterwards, samples were centrifuged at $17,000 \times g$ for $5 \mathrm{~min}$ and supernatants transferred to new tubes. Gross nucleic acid abundances were quantified using a Nanodrop instrument and $75 \mu \mathrm{g}$ chromatin mixed with RNAse inhibitor and $650 \mathrm{pmol}$ of oligonucleotides (OGNs) conjugated to Biotin-TEG. 
(Biotin-TEG increases OGN-biotin distances to $\sim 15$ atoms using a triethyleneglycol (TEG) spacer. Biotin-TEG therefore is used to avoid steric hindrances and improve attachment of OGNs to beads.) Samples were vortexed carefully and spun down, stored at $55^{\circ} \mathrm{C}$ and shaked on $600 \mathrm{rpm}$ for $10 \mathrm{~min}$. Temperature was decreased to room temperature and samples stored overnight at $55^{\circ} \mathrm{C}$. The beads were mixed with $1 \mathrm{ml}$ of Solution $1(1 \mathrm{M} \mathrm{NaCl}$ and $0.1 \%$ Tween), tubes vortexed, centrifuged shortly and transferred to a magnetic rack. The beads were washed $3 \times$ with solution 1 . In the meantime, samples were adjusted to $1 \mathrm{M} \mathrm{NaCl}$, vortexed and $50 \mu \mathrm{l}$ of beads and RNase inhibitor added to the solution. The tubes were stored on a rotator for $30 \mathrm{~min}$ at RT, centrifuged, stored in the magnetic rack and supernatants discarded. Beads were washed $2 \times$ with $1 \mathrm{ml}$ of Solution 1 and $3 \times$ with $1 \mathrm{ml}$ of Solution $2(1 \mathrm{M} \mathrm{NaCl}, 0.1 \%$ Tween, $0.1 \% \mathrm{SDS})$. For this, solutions were added, tubes vortexed and stored on a shaker $1050 \mathrm{rpm}$ set to $16^{\circ} \mathrm{C}$ for $5 \mathrm{~min}$. Afterwards, tubes were stored in the magnetic rack and supernatant discarded. For the final elution, beads were resuspended in $300 \mu \mathrm{l}$ elution buffer $(0.2 \mathrm{M} \mathrm{NaCl}, 0.1 \%$ SDS, $10 \mathrm{mM}$ Tris $\mathrm{pH}=8.0$ ) and $1 \times$ Proteinase inhibitor. The samples were incubated on a ThermoShaker for $4-5 \mathrm{~h}$ and shaken at $1450 \mathrm{rpm}$ at $65^{\circ} \mathrm{C}$. Finally, eluate was concentrated with Amicon Ultra- 0,5 Centrifugal filter. To degrade RNA contaminations, samples were treated with RNAse A. Sequences for $H 19$ sense and antisense oligonucleotides for RNA-immunoprecipitation are provided in Supplementary Data 6.

Sample preparation for MS. DTT was added at a final concentration of $5 \mathrm{mM}$ and samples were incubated on $55^{\circ} \mathrm{C}$ for $30 \mathrm{~min}$. Afterwards, samples were cooled down to room temperature and Cloroacetamid (CAA) added at final concentrations of $40 \mathrm{mM}$ for $30 \mathrm{~min}$ in the dark. Protein digestion was performed using the Single-Pot Solid-Phase-enhanced Sample Preparation technology (experimental details available upon request). In brief, $2 \mu \mathrm{L}$ of a $10 \mathrm{mg} / \mathrm{mL}$ mixture of hydrophilic and hydrophobic carboxylate coated paramagnetic beads (SeraMag Speed Beads, Cat No. 44152105050250 and 24152105050250 , GE Healthcare) were added to each sample. Acidified acetonitrile was added to achieve a final concentration of $50 \%$ organic solvent. Bound proteins were washed with $70 \%$ ethanol and $100 \%$ acetonitrile. Beads were resuspended in $5 \mu \mathrm{L} 50 \mathrm{mM}$ Triethylammoniumbicarbonate buffer containing $0.1 \mu \mathrm{g}$ Trypsin (Sigma) and $0.1 \mu \mathrm{g}$ LysC (Wako). Digestion was carried out for $16 \mathrm{~h}$ at $37^{\circ} \mathrm{C}$ in a PCR cycler to ensure constant temperatures. Recovered peptides were resuspended in $1 \%$ formic acid / 5\% DMSO and stored at $-20^{\circ} \mathrm{C}$ prior to $\mathrm{MS}$ analysis.

Mass spectrometry. All samples were analyzed on a Q-Exactive Plus (Thermo Scientific) mass spectrometer that was coupled to an EASY nLC 1000 UPLC (Thermo Scientific). Peptides were loaded with solvent A ( $0.1 \%$ formic acid in water) onto an in-house packed analytical column $(50 \mathrm{~cm} \times 75 \mu \mathrm{m}$ I.D., filled with $2.7 \mu \mathrm{m}$ Poroshell EC120 C18, Agilent). Peptides were chromatographically separated at a constant flow rate of $250 \mathrm{~nL} / \mathrm{min}$ using the following gradient: $5-30 \%$ solvent B (0.1\% formic acid in $80 \%$ acetonitrile) within $119 \mathrm{~min}, 30-50 \%$ solvent B within $19 \mathrm{~min}$, followed by washing and column equilibration. The mass spectrometer was operated in data-dependent acquisition mode. The MS1 survey scan was acquired from $300-1750 \mathrm{~m} / \mathrm{z}$ at a resolution of 70,000 . The top ten most abundant peptides were isolated within a $2 \mathrm{Da}$ window and subjected to HCD fragmentation at a normalized collision energy of $27 \%$. The AGC target was set to $5 \times 10 \mathrm{E} 5$ charges, allowing a maximum injection time of $55 \mathrm{~ms}$. Product ions were detected in the Orbitrap at a resolution of 17,500 . Precursors were dynamically excluded for $20 \mathrm{~s}$.

Bioinformatic analysis of MS data. All mass spectrometric raw data were processed with Maxquant (version 1.5.3.8) using default parameters. Briefly,

MS2 spectra were searched against the Uniprot MOUSE.fasta database, including a list of common contaminants. False discovery rates on protein and PSM level were estimated by the target-decoy approach to $0.01 \%$ (Protein FDR) and $0.01 \%$ (PSM FDR). Minimal peptide length was set to 7 amino acids and carbamidomethyolation at cysteine residues was considered as a fixed modification. Oxidation (M) and Acetyl (Protein N-term) were included as variable modifications. The matchbetween runs option was enabled. LFQ quantification was enabled using default settings. The Maxquant output was processed as follows: protein groups flagged as reverse, potential contaminant or only identified by site were removed from the proteinGroups.txt. The remaining table was analyzed with R. Protein groups with at least two valid values out of three replicates (or 2 out of 2 ) in at least one of the bait subgroups were directed to statistical analysis. The R STATS Package was used to perform Student's t-tests to obtain significantly changed proteins. Those peptides with $p$-values $<0.05$ and $\log 2$ fold-changes $>1$ were considered as signficantly different. Proteins that provide 2 or 3 valid values in one bait group and zero in the other, were also considered as significantly different.

Ingenuity pathway analysis and AMIGO2 GO Term Analysis. In total 3590 peptides were detected by MS. All 168 uniquely identified and significantly enriched (see significance criteria above) peptides with fold-changes $\geq 1.5$ between $H 19$ antisense compared to sense oligonucleotide immunoprecipitation in confluent $(n=114)$ or differentiated $(n=102)$ conditions were analyzed using Ingenuity Pathway Analysis. To construct a functionally interconnected $H 19$ protein interactome, all $n=168$ gene were loaded into IPA and gene nodes with $n \geq 1$ edges to other genes retained. For GO Term classification of the remaining $n=61 \mathrm{H} 19$ interaction partners, corresponding Gene Symbols were loaded into AMIGO2 (http://amigo.geneontology. org) and those proteins counted classified by GO Terms RNA binding or Chromatin binding or Chromating modification.

Analyses of public datasets. PEG and MEG expression in WAT of 24 strains of mice was extracted from publicly available datasets as described by Kraus et al. ${ }^{65}$, Briefly, expression levels of PEGs and MEGs in WAT were obtained from [www. BioGPS.org] - Adipose (MOE430 V2) Obesity-resistant versus obesity-prone is defined based on body composition data from Svenson et al. ${ }^{66}$ and "Naggert1" in the Mouse Phenome Database [www.jax.org/phnome], in which body composition was measured in 43 different mouse stains that were fed a high-fat diet for 8 weeks. Mouse line PL/J is not studied in Naggert1, but its percentage fat weight at 20 months of age is approximately one standard deviation below the average of 32 mouse strains in the Ackert1 data set [www.jax.org/phnome]. PEG and MEG expression was analyzed by GSEA in surgically removed biopsies of WAT of future high and low weight gainers from C57BL6/J mice biopsied prior to treatment with high-fat $\operatorname{diet}^{53}$.

Serum analyses. Serum was obtained by allowing the blood to clot at $4{ }^{\circ} \mathrm{C}$ for $4 \mathrm{~h}$ The clotted blood was centrifuged for $10 \mathrm{~min}$ at $6200 \times \mathrm{g}$, the supernatants transferred to a new tube and centrifuged for another $10 \mathrm{~min}$ at $17,000 \times \mathrm{g}$. Serum was collected and stored at $-80^{\circ} \mathrm{C}$ diluted $1: 3$ with $0.9 \% \mathrm{NaCl}$. For analysis of cholesterole levels the Cholesterin CHOD-PAP (Roche Diagnostics) and to determine trigycerides levels the Triglyceride GPO-PAP of Roche Diagnostics was used. Both analyses were run with the Cobas C 702 (Roche Diagnostics). The analysis was conducted at the Institute for clinical chemistry at the University Clinics in Cologne.

Pyrosequencing. DNA methylation was analyzed by pyrosequencing. To this end, $1 \mu \mathrm{g}$ DNA from $1^{\circ} \mathrm{BAT}$ transfected with siCtrl or siH19 was Bisulfite-converted using the EZ DNA Methylation kit (Zymo), PCR amplified, sequenced on a PyroMark Q96 (Qiagen), and analyzed with the PyroMark CpG SW 1.0 software (Qiagen) as described recently ${ }^{67}$. Primer sequences are provided in Supplementary Data 6.

\section{Data availability}

All relevant data are available from the authors. Raw data were deposited within GEO under accession no. GSE116227.

Received: 17 December 2017 Accepted: 31 July 2018

Published online: 06 September 2018

\section{References}

1. Van Gaal, L. F., Mertens, I. L. \& De Block, C. E. Mechanisms linking obesity with cardiovascular disease. Nature 444, 875-880 (2006).

2. Hotamisligil, G. S. Endoplasmic reticulum stress and atherosclerosis. Nat. Med. 16, 396-399 (2010).

3. Torres, D. M., Williams, C. D. \& Harrison, S. A. Features, diagnosis, and treatment of nonalcoholic fatty liver disease. Clin. Gastroenterol. Hepatol. 10, 837-858 (2012).

4. Bartelt, A. \& Heeren, J. The holy grail of metabolic disease: brown adipose tissue. Curr. Opin. Lipidol. 23, 190-195 (2012).

5. van Marken Lichtenbelt, W. D. et al. Cold-activated brown adipose tissue in healthy men. N. Engl. J. Med. 360, 1500-1508 (2009).

6. Feldmann, H. M., Golozoubova, V., Cannon, B. \& Nedergaard, J. UCP1 ablation induces obesity and abolishes diet-induced thermogenesis in mice exempt from thermal stress by living at thermoneutrality. Cell. Metab. 9, 203-209 (2009).

7. Yoneshiro, T. et al. Recruited brown adipose tissue as an antiobesity agent in humans. J. Clin. Invest. 123, 3404-3408 (2013).

8. Hanssen, M. J. et al. Short-term cold acclimation improves insulin sensitivity in patients with type 2 diabetes mellitus. Nat. Med. 21, 863-865 (2015).

9. Kajimura, S., Seale, P. \& Spiegelman, B. M. Transcriptional control of brown fat development. Cell. Metab. 11, 257-262 (2010).

10. Birney, E. et al. Identification and analysis of functional elements in $1 \%$ of the human genome by the ENCODE pilot project. Nature 447, 799-816 (2007).

11. Derrien, T. et al. The GENCODE v7 catalog of human long noncoding RNAs analysis of their gene structure, evolution, and expression. Genome Res. 22, $1775-1789$ (2012)

12. Ulitsky, I. \& Bartel, D. P. lincRNAs: genomics, evolution, and mechanisms. Cell 154, 26-46 (2013). 
13. Iyer, M. K. et al. The landscape of long noncoding RNAs in the human transcriptome. Nat. Genet. 47, 199-208 (2015).

14. Kim, J. et al. Long noncoding RNAs in diseases of aging. Biochim. Biophys. Acta 1859, 209-221 (2016)

15. Kornfeld, J. W. \& Bruning, J. C. Regulation of metabolism by long, noncoding RNAs. Front. Genet. 5, 57 (2014).

16. Mi, L., Zhao, X. Y., Li, S., Yang, G. \& Lin, J. D. Conserved function of the long noncoding RNA Blncl in brown adipocyte differentiation. Mol. Metab. 6, 101-110 (2017).

17. You, L. et al. GM13133 is a negative regulator in mouse white adipocytes differentiation and drives the characteristics of brown adipocytes. J. Cell. Physiol. 233, 313-324 (2018).

18. van Heesch, S. et al. Extensive localization of long noncoding RNAs to the cytosol and mono- and polyribosomal complexes. Genome Biol. 15, R6 (2014).

19. Cohen, P. \& Spiegelman, B. M. Brown and beige fat: molecular parts of a thermogenic machine. Diabetes 64, 2346-2351 (2015).

20. Bartolomei, M. S., Zemel, S. \& Tilghman, S. M. Parental imprinting of the mouse H19 gene. Nature 351, 153-155 (1991).

21. Bell, A. C. \& Felsenfeld, G. Methylation of a CTCF-dependent boundary controls imprinted expression of the Igf2 gene. Nature 405, 482-485 (2000).

22. Boucher, J. et al. Insulin and insulin-like growth factor 1 receptors are required for normal expression of imprinted genes. Proc. Natl Acad. Sci. USA 111, 14512-14517 (2014)

23. Dey, B. K., Pfeifer, K. \& Dutta, A. The H19 long noncoding RNA gives rise to microRNAs miR-675-3p and miR-675-5p to promote skeletal muscle differentiation and regeneration. Genes Dev. 28, 491-501 (2014).

24. Cristancho, A. G. et al. Repressor transcription factor 7-like 1 promotes adipogenic competency in precursor cells. Proc. Natl Acad. Sci. USA 108, 16271-16276 (2011).

25. Alvarez-Dominguez, J. R. et al. De novo reconstruction of adipose tissue transcriptomes reveals long non-coding RNA regulators of brown adipocyte development. Cell. Metab. 21, 764-776 (2015).

26. Tseng, Y. H. et al. Prediction of preadipocyte differentiation by gene expression reveals role of insulin receptor substrates and necdin. Nat. Cell Biol. 7, 601-611 (2005)

27. Subramanian, A., Kuehn, H., Gould, J., Tamayo, P. \& Mesirov, J. P. GSEA-P: a desktop application for Gene Set Enrichment Analysis. Bioinformatics 23 3251-3253 (2007).

28. Guerra, C. et al. Brown adipose tissue-specific insulin receptor knockout shows diabetic phenotype without insulin resistance. J. Clin. Invest. 108, 1205-1213 (2001).

29. Oliverio, M. et al. Dicer1-miR-328-Bace1 signalling controls brown adipose tissue differentiation and function. Nat. Cell Biol. 18, 328-336 (2016).

30. Schlein, C. \& Heeren, J. Implications of thermogenic adipose tissues for metabolic health. Best. Pract. Res. Clin. Endocrinol. Metab. 30, 487-496 (2016).

31. Ainscough, J. F., Koide, T., Tada, M., Barton, S. \& Surani, M. A. Imprinting of Igf2 and $\mathrm{H} 19$ from a $130 \mathrm{~kb}$ YAC transgene. Development 124, 3621-3632 (1997).

32. Smits, G. et al. Conservation of the H19 noncoding RNA and H19-IGF2 imprinting mechanism in therians. Nat. Genet. 40, 971-976 (2008).

33. Vernochet, C. et al. Adipose tissue mitochondrial dysfunction triggers a lipodystrophic syndrome with insulin resistance, hepatosteatosis, and cardiovascular complications. FASEB J 28, 4408-4419 (2014).

34. Boutant, M. et al. Mfn2 is critical for brown adipose tissue thermogenic function. EMBO J 36, 1543-1558 (2017).

35. Barlow, D. P., Stoger, R., Herrmann, B. G., Saito, K. \& Schweifer, N. The mouse insulin-like growth factor type-2 receptor is imprinted and closely linked to the Tme locus. Nature 349, 84-87 (1991).

36. Morison, I. M., Ramsay, J. P. \& Spencer, H. G. A census of mammalian imprinting. Trends Genet. 21, 457-465 (2005).

37. Kong, A. et al. Parental origin of sequence variants associated with complex diseases. Nature 462, 868-874 (2009).

38. Wakeling, E. L. et al. Diagnosis and management of Silver-Russell syndrome: first international consensus statement. Nat. Rev. Endocrinol. 13, 105-124 (2017).

39. Hark, A. T. et al. CTCF mediates methylation-sensitive enhancer-blocking activity at the H19/Igf2 locus. Nature 405, 486-489 (2000).

40. Ferguson-Smith, A. C. Genomic imprinting: the emergence of an epigenetic paradigm. Nat. Rev. Genet. 12, 565-575 (2011).

41. Meng, L. et al. Towards a therapy for Angelman syndrome by targeting a long non-coding RNA. Nature 518, 409-412 (2015).

42. Moore, T. \& Haig, D. Genomic imprinting in mammalian development: a parental tug-of-war. Trends Genet. 7, 45-49 (1991).

43. Peters, J. The role of genomic imprinting in biology and disease: an expanding view. Nat. Rev. Genet. 15, 517-530 (2014).
44. Al Adhami, H. et al. A systems-level approach to parental genomic imprinting: the imprinted gene network includes extracellular matrix genes and regulates cell cycle exit and differentiation. Genome Res. 25, 353-367 (2015).

45. Varrault, A. et al. Zacl regulates an imprinted gene network critically involved in the control of embryonic growth. Dev. Cell. 11, 711-722 (2006).

46. Berg, J. S. et al. Imprinted genes that regulate early mammalian growth are coexpressed in somatic stem cells. PLoS ONE 6, e26410 (2011).

47. Dalgaard, K. et al. Trim 28 haploinsufficiency triggers bi-stable epigenetic obesity. Cell 164, 353-364 (2016)

48. Gabory, A. et al. H19 acts as a trans regulator of the imprinted gene network controlling growth in mice. Development 136, 3413-3421 (2009).

49. Martinet, C. et al. H19 controls reactivation of the imprinted gene network during muscle regeneration. Development 143, 962-971 (2016).

50. Monnier, P. et al. H19 lncRNA controls gene expression of the Imprinted Gene Network by recruiting MBD1. Proc. Natl Acad. Sci. USA 110, 20693-20698 (2013)

51. Simon, M. D. et al. The genomic binding sites of a noncoding RNA. Proc. Natl Acad. Sci. USA 108, 20497-20502 (2011).

52. Liao, B., Hu, Y., Herrick, D. J. \& Brewer, G. The RNA-binding protein IMP-3 is a translational activator of insulin-like growth factor II leader-3 mRNA during proliferation of human K562 leukemia cells. J. Biol. Chem. 280 18517-18524 (2005).

53. Koza, R. A. et al. Changes in gene expression foreshadow diet-induced obesity in genetically identical mice. PLoS Genet. 2, e81 (2006).

54. Wu, C. et al. BioGPS: an extensible and customizable portal for querying and organizing gene annotation resources. Genome Biol. 10, R130 (2009).

55. Kamei, Y. et al. Peg1/Mest in obese adipose tissue is expressed from the paternal allele in an isoform-specific manner. FEBS Lett. 581, 91-96 (2007).

56. Takahashi, M., Kamei, Y. \& Ezaki, O. Mest/Peg1 imprinted gene enlarges adipocytes and is a marker of adipocyte size. Am. J. Physiol. Endocrinol. Metab. 288, E117-E124 (2005).

57. Haig, D. Genomic imprinting and kinship: how good is the evidence? Annu. Rev. Genet. 38, 553-585 (2004).

58. Kotzbeck, P. et al. Brown adipose tissue whitening leads to brown adipocyte death and adipose tissue inflammation. J. Lipid Res. 59, 784-794 (2018).

59. Thorvaldsen, J. L., Fedoriw, A. M., Nguyen, S. \& Bartolomei, M. S. Developmental profile of H19 differentially methylated domain (DMD) deletion alleles reveals multiple roles of the DMD in regulating allelic expression and DNA methylation at the imprinted H19/Igf2 locus. Mol. Cell. Biol. 26, 1245-1258 (2006).

60. Wagle, P., Nikolic, M. \& Frommolt, P. QuickNGS elevates next-generation sequencing data analysis to a new level of automation. BMC Genom. 16, 487 (2015).

61. Klein, J. et al. beta(3)-adrenergic stimulation differentially inhibits insulin signaling and decreases insulin-induced glucose uptake in brown adipocytes. J. Biol. Chem. 274, 34795-34802 (1999).

62. Brand, M. D. \& Nicholls, D. G. Assessing mitochondrial dysfunction in cells Biochem. J. 435, 297-312 (2011).

63. Gao, J. et al. CLUH regulates mitochondrial biogenesis by binding mRNAs of nuclear-encoded mitochondrial proteins. J. Cell. Biol. 207, 213-223 (2014).

64. Kloting, N. et al. Insulin-sensitive obesity. Am. J. Physiol. Endocrinol. Metab. 299, E506-E515 (2010)

65. Kraus, D. et al. Nicotinamide N-methyltransferase knockdown protects against diet-induced obesity. Nature 508, 258-262 (2014)

66. Svenson, K. L. et al. Multiple trait measurements in 43 inbred mouse strains capture the phenotypic diversity characteristic of human populations. J. Appl. Physiol. (1985) 102, 2369-2378 (2007)

67. Weidner, C. I. et al. Aging of blood can be tracked by DNA methylation changes at just three CpG sites. Genome Biol. 15, R24 (2014).

\section{Acknowledgements}

We thank Christiane Schäfer and Pia Scholl for HE stainings and Beatrix Martiny for electron microscopy and Julia Husa and Markus Jeitler for help with cell culture and RNA-Seq. We acknowledge Jens Alber for technical assistance. Jenny Blommer determined TG and total cholesterole levels. Linheng Li from the Stowers Institute provided H19-DMR flDMR/flDMR mice. We thank Christian Frese, Brigitte Kisters-Woike, and Corinna Klein from CECAD Proteomics Core Facility. J.W.K., E.S., I.G., N.H., S.K., and M.O. are supported by the Emmy-Noether Program of the Deutsche Forschungsgemeinschaft (DFG; KO4728/1.1). J-W.K. receives funding from University of Southern Denmark (SDU) and Danish Diabetes Academy (DDA), which is funded by Novo Nordisk Fonden (NNF). M.P.J is grateful for support by CECAD. R.L., E.F-R., M.P.J., and P.K. receive support from the European Research Council (ERC) Starting Grant TransGenRNA (No. 675014). E.S. is supported by Evangelisches Studienwerk 
Villigst. S.K appreciates support from DAAD. N.R.H. received a stipend from the Cologne Graduate School for Ageing (CGA). E.N. was supported by an Alexander-vonHumboldt postdoctoral fellowship. This work was supported by the DFG, Obesity Mechanisms (SFB 1052, B01) to M.B.

\section{Authors contributions}

E.S., I.D., I.G., M.O., P.K., M.A., G.M., E.F.-R., P.H, M.P.J., R.L., N.R.H., S.K., M.H., and P.Z. performed the experiments, C.K. maintained experimental animals cohorts, E.N., U.A.Ø, J.H., W.W., and P.F. contributed discussions and performed training, and M.Blü., M.Bil., and J-W.K. conceived the experiments.

\section{Additional information}

Supplementary Information accompanies this paper at https://doi.org/10.1038/s41467018-05933-8.

Competing interests: The authors declare no competing interests.

Reprints and permission information is available online at http://npg.nature.com/ reprintsandpermissions/

Publisher's note: Springer Nature remains neutral with regard to jurisdictional claims in published maps and institutional affiliations.

(c) (i) adaptation, distribution and reproduction in any medium or format, as long as you give appropriate credit to the original author(s) and the source, provide a link to the Creative Commons license, and indicate if changes were made. The images or other third party material in this article are included in the article's Creative Commons license, unless indicated otherwise in a credit line to the material. If material is not included in the article's Creative Commons license and your intended use is not permitted by statutory regulation or exceeds the permitted use, you will need to obtain permission directly from the copyright holder. To view a copy of this license, visit http://creativecommons.org/ licenses/by/4.0/.

(c) The Author(s) 2018 